\begin{tabular}{|c|c|c|}
\hline Beitr. Ent. & Keltern & ISSN 0005-805X \\
\hline $\mathbf{5 7}(2007) 1$ & S. $9-36$ & 30.06 .2007 \\
\hline
\end{tabular}

\title{
Die Trauermücken in Deutschland - ökosystemare Bedeutung, zönologische Koinzidenzen und bioindikatorisches Potential
}

\section{(Diptera: Sciaridae)}

Mit 3 Figuren und 2 Tabellen

\author{
Frank Menzel und Ulrich Schulz
}

\begin{abstract}
Zusammenfassung
Trauermücken (Sciaridae) leisten mit über 400 bisher nachgewiesenen Arten in Deutschland einen wichtigen Beitrag zur Dipteren-Vielfalt in Natur- und Kulturlandschaften. Ihre ökosystemare Bedeutung ergibt sich aus der Lebensweise der Larven, die in hohen Abundanzen verschiedenste Böden besiedeln und hier als Phytosaprophage wichtige Zersetzerfunktionen übernehmen. Nach der Darstellung von Biodiversitäten bzw. Ökosystemleistungen wird eine Übersicht über die Artenzahlen in verschiedenen Biotopen Deutschlands gegeben. Die Zusammenfassung basiert auf allen bisher publizierten Daten zur Populationsdichte, Biomasse und Verbreitung der Trauermücken in Deutschland. Erstmals werden für jedes Biotop die dominanten Sciaridenarten im Sinne von zönologischen Koinzidenzen abgeleitet. Darauf basierend wird das mögliche Potential der Trauermücken als Indikatorgruppe diskutiert.
\end{abstract}

\section{Summary}

Black Fungus Gnats (Sciaridae), with more than 400 species recorded in Germany, contribute significantly to biodiversity of Diptera in natural and man-made landscapes. Their importance for the ecosystem is a result of the phytosaprophagous habits of the larvae, their function in the process of decomposition, and their frequent occurrence at high densities, in almost all types of soil. After the description of their contribution to biodiversity and the services which they render to the ecosystem, an overview of the number of species in different German biotopes is given. This synopsis is based on all published data on population densities, biomass and distribution of Sciarid flies in Germany. For the first time a survey is presented of Sciarid biodiversity and community structure (coincidences of dominant species) in different habitats. Based on this, the suitability of Sciaridae as bio-indicators is discussed.

Key words

Diptera, Nematocera, Sciaridae, Germany, biodiversity, distribution, ecology, soil dwelling larvae, ecosystem services, community structure, bio-indicators

\section{Einleitung}

Seit der UN-Konferenz für Umwelt und Entwicklung 1992 in Rio de Janeiro und ihren Nachfolgekonferenzen rücken Beiträge zur Biodiversität immer mehr in den Mittelpunkt des öffentlichen Interesses. Infolgedessen finden auch Untersuchungen zum Artenreichtum verschiedener Insektengruppen, die eine hohe Diversität besitzen und weit verbreitet sein können, in wissenschaftlichen Großprojekten ihren Niederschlag. Im Jahre 1993 hat auch die Bundesrepublik 
Deutschland das Übereinkommen über die biologische Vielfalt (Convention on Biological Diversity) unterzeichnet und sich u. a. dazu verpflichtet, die Artenvielfalt innerhalb der eigenen Landesgrenzen zu schützen (BUNR 2002). Seitdem ist man auch von offizieller Seite bemüht, mehr über die tatsächliche Formenvielfalt in den Lebensräumen Deutschlands zu erfahren, um anthropogene Auswirkungen auf die Flora und Fauna abschätzen zu können. Dies findet nicht nur in den Aktivitäten des Bundesamtes für Naturschutz seinen Niederschlag (KoRN \& VeIT 2003), sondern spiegelt sich europaweit auch in verschiedenen wissenschaftlichen Netzwerken mit deutscher Beteiligung wieder (z. B. FAUNA EUROPAEA - www.faunaeur.org).

Meist ist nur dem biologisch Interessierten bekannt, daß es auch in Deutschland noch Tiergruppen gibt, von denen die tatsächliche Artenvielfalt weitgehend unbekannt und die Ökologie der Arten nahezu unerforscht ist. Dazu gehören neben anderen Arthropodengruppen auch die Mücken (Nematocera) (LaSalle \& Gauld 1993, Schulz 2002 u. a.). In dieser Arbeit soll mit den Trauermücken (Sciaridae) eine Zweiflüglerfamilie betrachtet werden, die - ähnlich wie andere, eher bodenorientierte Dipteren - sehr individuenreich in Erscheinung tritt und die aus verschiedenen Gründen (v. a. Kleinheit der Objekte, präparative Hemmnisse) bei ökologischen Freilandarbeiten oft vernachlässigt wird (Hövemeyer 1991, Frouz 1999). Welches Potential diese Insektengruppe für zukünftige ökologische Studien haben kann, soll anhand verschiedener Aspekte verdeutlicht werden. Dabei wird das bisher publizierte Wissen zur Biologie, Ökologie und Verbreitung der Sciaridae in Deutschland zusammengefaßt und durch unpublizierte Informationen, Erfahrungen oder Schlußfolgerungen der Autoren ergänzt.

\section{Artenzahlen und Verteilungsmuster}

Bei kaum einer anderen Dipterengruppe gab es seit 1990 einen solchen Erkenntnisgewinn wie bei den Trauermücken. Ausgehend von einer ersten Faunenzusammenstellung für die ostdeutschen Bundesländer (Menzel et al. 1990) hat sich die Zahl der in Deutschland nachgewiesenen Arten im Zeitraum von nur 15 Jahren fast verdoppelt. Während Menzel auf der Basis einer stark überarbeiteten Nomenklatur und Systematik im Jahr 2000 schon 343 Sciaridenarten auflistet, hat sich die Zahl der bis heute registrierten Spezies auf über 400 erhöht (MenZel 2000, MenZel \& Heller in prep.). Hierbei muss angemerkt werden, dass der Wissenszuwachs nicht nur auf weitere artliche Erstnachweise für die Region zurückzuführen ist. Mitunter geht der Zuwachs noch auf das Entdecken von bisher unbeschriebenen Sciaridenspezies zurück, so dass jüngere Faunenerhebungen im ländlichen Raum - in hiesiger Auslegung nicht nur in Naturschutzgebieten und Biosphärenreservaten - für den europäischen Arten- und Biotopschutz Bedeutung haben können (Menzel \& Mohrig 1991, Sommer et al. 1994, Heller 2000, Menzel 2000, Menzel et al. 2003). Bei diesem rasanten Anstieg der Artenzahlen stellen Menzel et al. (2002) fest, dass sich etwa 150 Spezies nahezu flächendeckend über die gesamte Kulturlandschaft der Bundesrepublik nachweisen lassen. Sie schätzen ein, dass man für Deutschland wahrscheinlich erst 60 bis $70 \%$ des real vorhandenen Arteninventars erfasst hat. Darum sind die genannten Zahlen ein Indiz dafür, dass man bei den Sciariden noch weit von der vollständigen Kenntnis ihrer tatsächlichen Diversität entfernt ist und künftig noch eine umfangreiche Forschungsarbeit zu leisten sein wird.

Die räumliche Verteilung der nachgewiesenen Sciaridenarten über die Bundesländer ist zur Zeit noch sehr heterogen ausgeprägt, was sowohl mit der naturräumlichen Ausstattung als auch mit dem regional unterschiedlichen Durchforschungsgrad zusammenhängt. Folglich ist die Erhebungsdichte (Anzahl der Fundpunkte, Sammelintensität) z. B. im Raum Berlin/Brandenburg viel geringer als in den Bundesländern mit Mittel- und Hochgebirgsanteilen. Außerdem kom- 
men in den Gebirgsregionen mehrere Arten mit alpiner oder boreoalpiner Verbreitung hinzu, die im nordostdeutschen Tiefland fehlen. Trotz dieser Umstände unterscheidet sich die in Berlin/ Brandenburg ermittelte Artenzahl kaum von der anderer, recht gut bekannter Landesfaunen. Denn nur noch in Bayern, Schleswig-Holstein und Thüringen wurden mehr als 170 Sciaridenspezies festgestellt (Menzel et al. 2003). Erstaunlich hoch ist der Anteil jener Arten, die man trotz intensiver Bemühungen lediglich in sehr wenigen Bundesländern gefunden hat. So sind in der Checkliste von Berlin/Brandenburg (Menzel et al. 2003) zur Zeit 29 Spezies verzeichnet, für die es höchstens in zwei weiteren Bundesländern noch Fundbelege gibt. Acht andere Arten sind gegenwärtig sogar nur aus Berlin/Brandenburg bekannt, so dass sich daraus eine gesamtdeutsche Bedeutung ableiten lässt. Zu dieser Kategorie zählen die Arten Bradysia postrufescens MoHrIg \& Menzel, $B$. pseudodalmatina Mohrig \& Röschmann, B. subrufescens Mohrig \& Krivosheina, Claustropyga clausa (Tuomikoski), Corynoptera compressa (WALKer), Cratyna contracta MoHrig \& Röschmann, Epidapus debilis Menzel und Phytosciara producta Tuomikoski. Von den genannten Spezies liegen zudem nur sehr wenige Exemplare aus der gesamten Paläarktis vor. Mitunter existiert vom jeweiligen Taxon nur noch das Typenmaterial. Andere Funde sind in Mitteleuropa unikal oder darüber hinaus sogar für die europäische Fauna bedeutsam.

Nach gegenwärtigem Kenntnisstand sind ca. $80 \%$ aller Trauermücken-Arten feuchtigkeitsliebend und bevorzugen als Lebensraum beschattete Wälder, Moore oder Feuchtwiesen. Nach den Literaturrecherchen zur artlichen Verteilung der Sciariden auf ausgewählte Biotope in Deutschland fällt zunächst einmal auf, dass in den Wäldern offensichtlich ein höherer Artenreichtum verzeichnet wird als im Offenland oder anderen Lebensräumen (Tabelle 1). Wegen der verschiedenen Beprobungsintensität auf den Versuchsflächen und der Heterogenität der eingesetzten Fangmethoden darf man die herausgearbeiteten Zahlen jedoch nur vorsichtig vergleichen. Es scheint sich aber anzudeuten, dass in Nadelholzforsten weniger Arten vorkommen als in Laubholzwäldern. Diese Aussage ist offensichtlich nicht nur auf Reinbestände begrenzt, sondern trifft auch auf die bisher betrachteten Mischbestände zu. Dieser Trend geht sicher zu einem beträchtlichen Teil auf die mitunter gruppenspezifische Ernährungsweise der Larven zurück, was eine Einnischung in verschiedene Kleinstrukturen (z. B. verschiedene Substrate mit bestimmtem Mikroklima) zur Folge hat. Die Ursachen und funktionellen Aspekte der hohen Sciaridendiversität sind jedoch noch nicht aufgeklärt und können dem allgemeinen Phänomen zugeordnet werden, das schon Anderson (1975) als das „ungelöste Geheimnis der Diversität bodenlebender Tiere" bezeichnet hat.

Auch bezüglich der Funktionen hoher Artenzahlen ist noch vieles ungeklärt. So wird nach wie vor diskutiert, ob nur bestimmte „Schlüsselarten“ oder die Diversität aller Spezies für ÖkosystemFunktionen ausschlaggebend sind (z. B. Wolters 1998). Diese Fragestellung lässt sich auch auf Trauermücken-Lebensgemeinschaften übertragen. Siewerden oft von einer oder sehr wenigen Arten stark dominiert. Beispiele dafür sind: Sciara humeralis Zetterstedt für Moore (Heller 1998); Bradysia nitidicollis (MeIgen) und Cratyna nobilis (Winnertz) für das Feuchtgrünland (Heller 2000); Bradysia fungicola (WINNERTz) und Epidapus absconditus (VIMMER) für Erlenbruchwälder (Hövemeyer 1996a, Irmler et al. 1996); Bradysia confinis (Winnertz) und Corynoptera trepida (Winnertz) für Buchenwälder (Hövemeyer 1992a, Heller 1996); Ctenosciara hyalipennis (Meigen) für Kiefern- und Fichtenforste (Thiede 1977, Heller 1996); Corynoptera tridentata Hondru und Corynoptera perpusilla Winnertz für Halbtrockenrasen (Hövemeyer 1996b); Scatopsciara atomaria (Zetterstedt) für Ackerflächen (Froese 1992, Weber \& Prescher 1995, Heller 1996). Diese eudominanten Arten scheinen hauptsächlich die saprophagen Funktionen zu übernehmen, während andere Spezies möglicherweise hochspezialisiert sind, einer natürlichen Seltenheit unterliegen (Plachter 1991) oder stark auf anthropogene Nutzung reagieren. 


\section{Zur ökosystemaren Bedeutung der Sciaridae}

Allgemein hängt die funktionelle Bedeutung der Dipterentaxa für den Stoffkreislauf und den Energiefluß in einem Ökosystem von ihrer Abundanz und ihrer Biomasse ab (Brauns 1991, Hövemeyer 1992a, Frouz 1999). Im Stoffkreislauf der Wälder nehmen die Mückenlarven (Nematocera) grundsätzlich eine herausragende Stellung ein, weil sie v. a. im Boden sehr individuenreich vertreten sind (Fourman 1938, Zachariae 1965, Corssley 1977). Altmüller (1977) fand in Buchenwäldern mit Sauerhumus zeitweise 14.500 Zweiflüglerlarven in einem Quadratmeter Waldboden, von denen sich bis zu 98 \% saprophag ernähren (DunGER 1983). Er schätzt, dass allein die Larven der Sciaridae und Sciophilidae hier 13 bis $29 \%$ der jährlich anfallenden Laubstreu fressen. Auch andere Untersuchungen belegen die hohe Biomasse der Mückenlarven im Waldboden und deren Zersetzerleistung (Hövemeyer 1984, Weidemann \& Schauermann 1986, Feldmann 1991). Thiede (1977) fand z. B. mit Bodeneklektoren in Fichtenforsten des Solling heraus, dass auf einem Quadratmeter Waldboden ca. 1.000-2.600 Individuen schlüpfen können, wobei die Sciaridae neben den Cecidomyiidae (700-1.300 Individuen/ $\mathrm{m}^{2}$ ) die höchste Schlupfabundanz besaßen. Zudem betont Frouz (1999) in seinem Übersichtsartikel über bodenbesiedelnde Dipteren mehrmals, dass die Trauermücken (Sciaridae) zusammen mit den

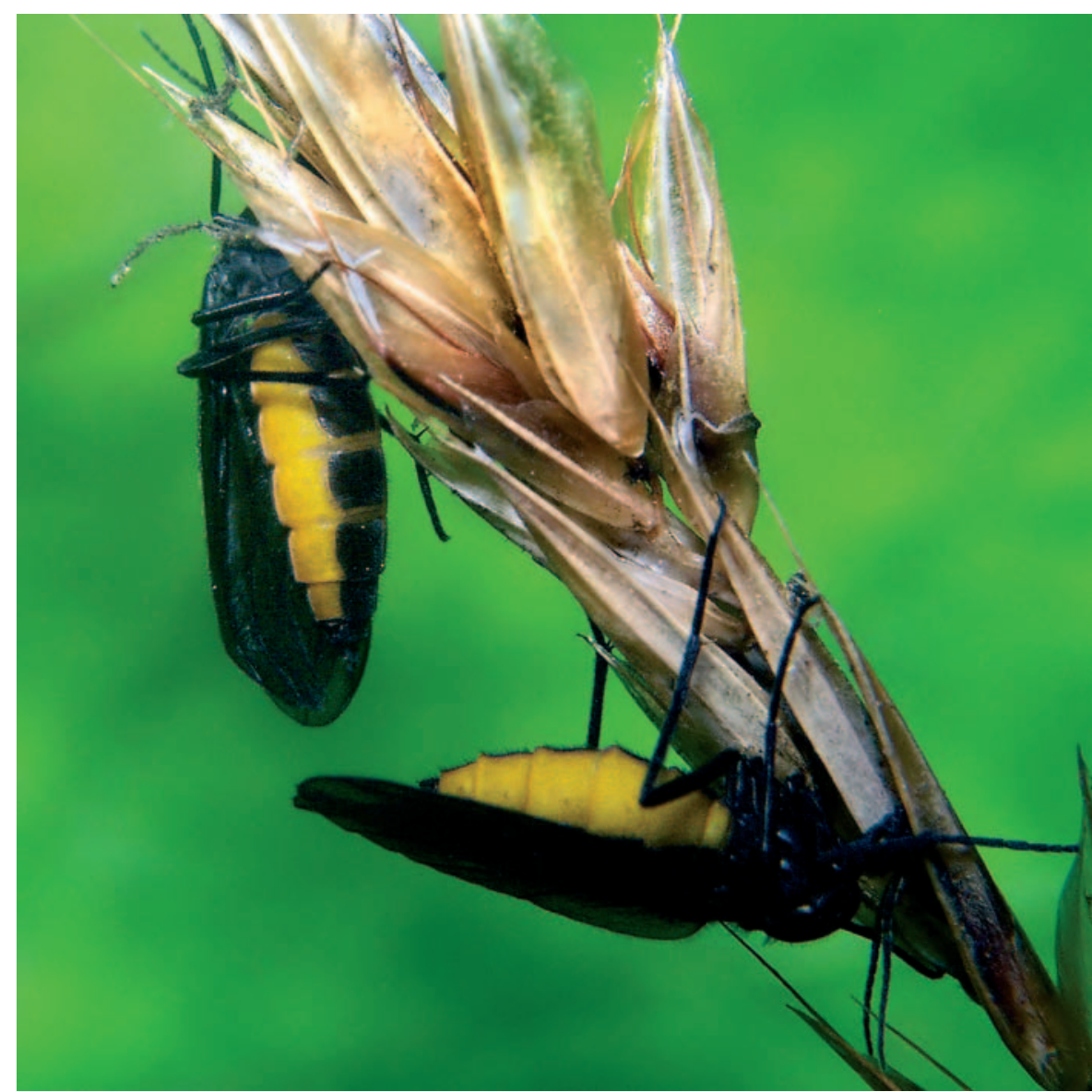

Fig. 1: Weibchen von Sciara analis Schiner an einem Süßgras-Blütenstand (Poaceae). Foto: F. KöHLER. 
Gallmücken (Cecidomyiidae) und Zuckmücken (Chironomidae) die höchsten Abundanzen in verschiedensten Ökosystemen aufweisen und dabei wichtige Aufgaben in der Streuzersetzung übernehmen.

Die ökosystemare Bedeutung der Trauermücken ergibt sich v. a. aus der zumeist phytosaprophagen Lebensweise der Larven. Im Waldboden gehören sie stets zu den dominierenden Insektenfamilien (Dunger 1983, Ellenberg et al. 1986). Nach Menzel \& Mohrig (2000) und anderen Autoren müssen ca. $75 \%$ der bis heute beschriebenen Sciaridenarten mit paläarktischer Verbreitung zur Gruppe mit phytosaprophagen Larven gezählt werden. HövemeYer (1984, 1985, 1987, 1989b, 1992a) wies bei seinen mehrjährigen Untersuchungen zur Struktur und Funktion der Bodenfauna in Rotbuchenwäldern nach, dass neben den Chironomiden und Cecidomyiiden die phytosaprophagen Sciaridenarten eine dominierende Stellung bei der Besiedlung der Bodenstreu durch Dipteren einnehmen. In den bodensauren Hainsimsen-Buchenwäldern des Solling gehörten $78 \%$ aller bodenlebenden Dipterenlarven zu den Trauermücken (AltmüLler 1977). Mit Abundanzwerten von 43 bis $46 \%$ [Larven] bzw. 35 bis $47 \%$ [Imagines] erwiesen sie sich auch in einem Buchenwald bei Göttingen als die mit Abstand häufigste Dipterengruppe (HövemeYer 1985). Einen wesentlichen Einfluss auf die Schlüpfabundanz der Sciaridenimagines haben v. a. die Streuschichtdicke, deren Feuchtigkeitsgehalt und Alter. Als Hauptaufenthaltsraum werden von den Larven der phytosaprophagen Sciaridenspezies offenbar die oberen Schichten des Waldbodens [ca. 2 bis $7 \mathrm{~cm}$ Tiefe] bevorzugt, wobei sie im Jahresverlauf erst zur Zeit des Laubfalls in die Streuschicht vordringen (Hövemeyer 1984, 1985). Außerdem ist bekannt, dass die Buchenstreu offensichtlich erst 2 bis 3 Jahre altern muss, bevor sie sich als Nahrung für Trauermückenlarven eignet (HövemeYer 1987, 1992b). Die Streu von anderen Laubgehölzen [z. B. Eiche] wird hingegen in kürzeren Zeiträumen zersetzt. Dabei können Änderungen in der Qualität der Bodenstreu offensichtlich positive oder negative Auswirkungen auf die Sciaridenzönose haben (PAQUIN \& Coderre 1997).

Bei Feldmann $(1991,1992)$ betrug der Anteil von Trauermücken in Bodenphotoeklektoren 47 bis $71 \%$ der gefangenen Dipteren - je nach dem, ob es sich um einen Kiefernjungbestand oder um einen älteren Mischbestand aus Rotbuche und Waldkiefer handelte. In Laubmischwäldern auf der fränkischen Alb machten die Trauermücken über $50 \%$ der erfassten Nematocera aus (Schulz 1996). Noch höhere Abundanzwerte bei den Sciaridenlarven erzielten Elmer \& Rотн (2001) in Abhängigkeit vom Alter des Baumbestandes sowie vom Umfang der BuchenBeimischung für Fichtenmonokulturen, Buchen-Fichten-Mischwälder und reine Buchenwälder im Erzgebirge. Hier stellten die Trauermücken durchschnittlich $62 \%$ der erfassten Dipterenlarven
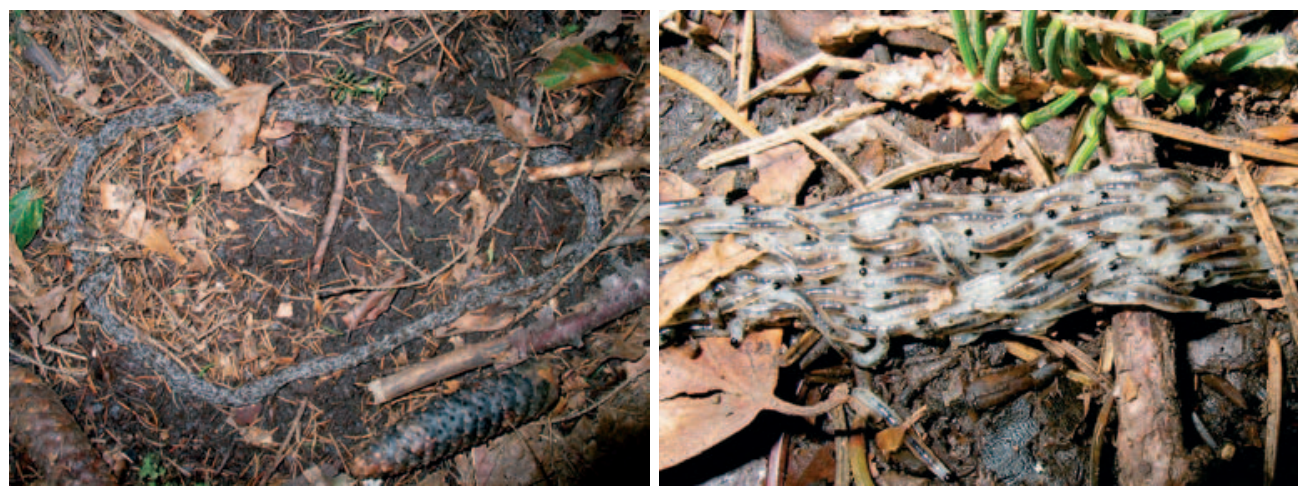

Fig. 2-3: Kleiner Larvenzug von Trauermücken (Diptera: Sciaridae) am 12. August 2005 im Nationalpark Bayerischer Wald (Niederbayern) - im Volksmund auch Heerwurm genannt. Fotos: C. Stierstorfer. 
[ca. $45 \%$ in Buchenbeständen bis $85 \%$ in Fichtenforsten]. Auch auf Halbtrockenrasen können die Trauermücken mit Dominanzwerten von 37 bis $74 \%$ die häufigste Dipterengruppe sein (HÖVEMEYer 1989c, 1996b).

Manchmal treten die Larven der Sciariden auf spektakuläre Weise in Erscheinung. So wird seit 1603 immer wieder von großen Larvenaggregationen in europäischen Laub- und Nadelwäldern berichtet, die nach vorausgegangener Massenentwicklung auf engstem Raum entstehen. Sie werden im deutschsprachigen Raum meist als „Heerwurm“ bezeichnet und können bis zu 14 Meter lange Wanderzüge bilden, die sich nicht selten aus bis zu 250.000 Sciaridenlarven zusammensetzen. Auf der Basis gezüchteter Materialien und gut illustrierter Beschreibungen stellten Menzel \& Mohrig (2000) heraus, dass nach gegenwärtigem Kenntnisstand nicht nur eine, sondern mehrere Sciaridenarten für das Phänomen verantwortlich sind. Namentlich gehören die Sciaridenspezies Bradysia bicolor (MeIgen), Cratyna perplexa (Winnertz), Ctenosciara hyalipennis (MeIgen), Sciara analis Schiner, Sciara hemerobioides (Scopoli) und Sciara militaris Nowicki zu den Heerwurmbildnern. In Deutschland wurde der „Heerwurm“ v. a. in den Mittelgebirgen gefunden (Menzel \& Mohrig 2000; Sacher 2001; Markel 2002a, b; Roth 2005).

Die zur Zeit nördlichste Fundmeldung in Deutschland stammt von Borrmann (2004), der einen Larvenzug im Naturschutzgebiet „Heilige Hallen“ bei Feldberg (Mecklenburg-Vorpommern) beobachtete. Eine umfangreiche Zusammenfassung mit weiterführenden Literaturquellen über den „Heerwurm“ - einschließlich der bis zum Jahr 2000 bekanntgewordenen Fundorte in Europa - geben Menzel \& Mohrig (2000).

Die Position der Dipterentaxa im Nahrungsnetz hängt maßgeblich von ihrer Ernährungsform ab (Hövemeyer 1992a, Frouz 1999, Brauns 1991). Weil die Imagines der Trauermücken keine Nahrung mehr aufnehmen, wird die Stellung der Sciaridenspezies von der Ernährungsweise der Larven vorgegeben. Als Bewohner meist sehr humusreicher Böden und als Primärverwerter von Faulstoffen sind die Trauermückenlarven ein unverzichtbarer Bestandteil in den Stoffkreisläufen der Natur und in den Nahrungsketten. Damit übernehmen sie wichtige Ökosystem-Funktionen [„,ecosystem services“]. Die Larven der Trauermücken haben oft auch einen erheblichen Einfluss auf die Bodenstruktur und die Bodenqualität. Ob sie deshalb nach der Definition von Lavelle et al. (1997) zusätzlich als Ökosystem-Ingenieure [„soil ecosystem engineers“] einzustufen sind, ist noch nicht ausreichend erforscht. HövemeYer (1989b) hat die ökosystemare Bedeutung der Trauermücken untersucht und spricht sogar davon, dass die Sciaridenlarven in ungestörten Laubwäldern dafür Sorge tragen, dass auch längerfristig von der Makrofauna nicht genutzte Laubstreu doch wieder in den Stoffkreislauf eingespeist werden kann.

Aufgrund der hohen Reproduktionsrate und der daraus resultierenden Individuenzahlen, die sie hervorbringen können, sind die Trauermücken auch als Beutetiere bedeutsam. Dies gilt sowohl für die Larven, als auch für die Imagines (KüHNE et al. 2004). Die adulten Trauermücken nehmen einen festen Platz im Nahrungsgefüge ein, weil sie u. a. von räuberischen Nematoden und Insekten, Spinnentieren, Amphibien, Eidechsen und Vögeln konsumiert werden (u. a. MenzeL \& Mohrig 2000). Für das stark ausgeprägte „Prädatorennetz“ des Waldbodens (SchauermanN 1976) sind sie eine wichtige Nahrungsquelle. Darauf deuten auch Manipulationsversuche von Schulz (1996) an Waldstreu hin.

Zusammenfassend lässt sich feststellen, dass die Trauermücken aufgrund ihrer ungewöhnlich hohen Individuenzahlen wichtige ökosystemare Funktionen ausüben. Im Vordergrund stehen dabei die „ecosystem services“, die von der überwiegend saprophagen Lebensweise der Sciaridenlarven bestimmt werden. 


\section{Ableitung zönologischer Koinzidenzen}

Zukünftige ökologische Forschungsprojekte erfordern eine Aufbereitung der bis heute publizierten Daten und eine übersichtlichere Darstellung des bisherigen Wissens über Sciaridenzönosen in Deutschland. Deshalb wird an dieser Stelle erstmals versucht, eine Übersicht für die Sciaridenarten mit hoher Nachweisstetigkeit zu geben. Für die betrachteten Biotoptypen werden jene Spezies herausgestellt, die in den betrachteten Strukturen dominant auftraten. Alle Informationen aus dem verfügbaren Literaturfundus werden in Tabelle 2 unkommentiert zusammengestellt. Dabei handelt es sich um einen ersten Versuch, für die Trauermückenfauna in Deutschland zönologische Koinzidenzen abzuleiten. Nach Kratochwil \& Schwabe (2001) versteht man darunter das gemeinsame Vorkommen einzelner Arten oder Artengruppen in bestimmten Lebensräumen.

Die Zusammenstellung (Tabelle 2) basiert auf den im Literaturverzeichnis aufgelisteten Quellen. In die Betrachtung wurden nur veröffentlichte Informationen zur Ökologie und Verbreitung von Trauermücken einbezogen, die auf dem Staatsgebiet Deutschlands erzielt wurden. Fehlbestimmungen oder artliche Fehldeutungen, die in den Publikationen feststellbar waren, wurden in Tabelle 2 kommentarlos berichtigt, weil diese mit den Literaturzitationen in MenzeL \& Mohrig (2000) bereits korrigiert worden sind. Aus diesem Grund können die hier zitierten validen Artnamen von den in der Originalschrift verwendeten Namen abweichen. Die nomenklatorische Richtigstellung [Verwendung valider Artnamen; richtige Positionierung der Spezies im System der Sciaridae] und die korrekte Zuordnung der damit verknüpften Informationen werden als wichtiger Bestandteil der vorliegenden Arbeit angesehen. Die Übersicht fasst lediglich das bisher bekannte Wissen zusammen, das durch zielgerichtete Freilanderhebungen - möglichst durch Langzeitstudien - ergänzt oder präzisiert werden muss.

Die Übersicht (Tabelle 1) zeigt, dass sich die ökologischen Erhebungen über Trauermücken in Deutschland und die daraus resultierenden Primärdaten ungleichmäßig auf die betrachteten Lebensräume verteilen. So existieren für manche Biotoptypen [z. B. Streuobstwiesen, Mischwälder] nur singuläre Faunenaufnahmen mit relativ kurzen Versuchszeiten. Im Gegensatz dazu zeichnen sich jene Untersuchungen, die z. B. auf Mooren, Ruderalfluren oder dem Grünland durchgeführt wurden, durch eine höhere Anzahl einbezogener Versuchsflächen und/oder durch partiell höhere Beprobungszeiten aus. Für andere Biotoptypen [z. B. Äcker, Rotbuchen- und Fichtenwälder] ergibt sich eine deutlich bessere Datenlage, weil diese schon mehrfach untersucht worden sind. Welchen Einfluss die Versuchsdauer und/oder die Anzahl der einbezogenen Flächen auf den Nachweis von Sciaridenspezies haben kann, wird durch die herausgestellten Artenzahlen verdeutlicht. Danach führt nur eine richtige Auswahl beider Parameter und deren effektive Kombination zu einem objektiven Forschungsergebnis. Weitere Unsicherheiten ergeben sich aus der methodischen Herangehensweise, die sich meist in der verwendeten Fangmethode und/oder der unterschiedlichen Zahl der ausgebrachten Fallen niederschlägt. Bei manchen Untersuchungen wurden z. B. nur Gelbschalen [GES] (Metzner \& Menzel 1996; Rudzinski 1992b, 1994), ausschließlich Malaisefallen [MAF] (Heller 1998, 1999) oder nur Bodenphotoeklektoren [BOE] (Heller 1990, 1996, 2002b; Hövemeyer 1992a, 1997; RudZINSKI 1989a, 1989b, 1992a; SCHRANKEL 1998) eingesetzt. Recht häufig sind jedoch die genannten Fallentypen untereinander, mit der Netzkescherstreifmethode [KEF] oder anderen Fallentypen kombiniert worden (z. B. FELDMANn 1991, 1992; Fritz 1982, 1983; Froese 1992; Heller 2000, 2002a; Hennicke et al. 1997; Holstein 1990; Hövemeyer 1996a; Leuckefeld 1995; Menzel \& Mohrig 1991; Schulz 1996; Werner 1997). Erwähnenswert sind hier v. a. Barberfallen [BAF], Stamm- [STE] und Wurzeleklektoren [WTE] sowie die Probenentnahme mit dem Stechrahmen [STR]. Zudem können sich die verwendeten Geräte, die auf dem gleichen Fangprinzip beruhen, in ihrer Konstruktion wesentlich voneinander unterscheiden [z. B. Gefäßdurchmesser, Fallenhöhe und/oder -färbung bei Barberfallen und Farbschalen]. Dazu 
muss man auch konstruktiv abgewandelte Eklektoren, Malaisefallen oder Kescher rechnen, die aufgabenspezifisch an verschiedene Raummaße, standortbezogene Gegebenheiten oder bestimmte Pflanzenteile adaptiert sind. Andererseits beeinflussen auch die Flächenexposition, witterungsbedingte Faktoren oder andere methodische Unschärfen [z. B. Leerungsrhythmen, Standzeiten im Jahresverlauf] das Fangergebnis. Infolgedessen können gravierende methodische Differenzen oder nicht vergleichbare Umweltdaten zu verschiedenen Ergebnissen, Aussagen und Interpretationen führen. Im Extremfall wären zwei Versuchsflächen mit ähnlicher Biotopausstattung schwer vergleichbar, weil die erzielten Fangdaten eine ganz andere, nicht in Übereinstimmung zu bringende Zusammensetzung der beiden Sciaridenzönosen vortäuschen. Hinzu kommt noch die mitunter recht grobe Einteilung der Biotoptypen, in der z. B. die Waldgerste-Buchenwälder und HainsimsenBuchenwälder unter dem Begriff „Rotbuchenwälder“ zusammengefasst sind. Solche Fehlerquellen müssen zu einem späteren Zeitpunkt beseitigt werden. Trotz der aufgezeigten Mängel können die vorgestellten Übersichten als erste Arbeitsgrundlage dienen und bei der methodischen Planung von Freilanderhebungen behilflich sein. Zudem lassen sich daraus neue Fragestellungen ableiten, die einen Beitrag zur Erforschung der Ökologie der Trauermücken leisten.

\section{Zum bioindikatorischen Potential der Trauermücken}

Dipteren gehören zu den artenreichsten Arthropodengruppen in Landökosystemen. Trotzdem haben sich bisher nur die Schwebfliegen (Syrphidae) als Indikatorgruppe im weiteren Sinne etabliert. Viele Dipterengruppen finden wegen ihrer schwierigen Erfaßbarkeit und aufgrund großer Probleme bei der Determination wenig Beachtung (Kratochwil \& Schwabe 2001). Bei ihnen werden jedoch bioindikatorische Potentiale vermutet und auch international wird gefordert, die Dipteren künftig verstärkt bei der Beurteilung von Lebensraumqualitäten [z. B. in Umweltgutachten] einzusetzen (HASLETT 2001).

Die Familie der Sciaridae eignet sich aufgrund der erreichten Forschungsergebnisse v. a. in Deutschland für Langzeituntersuchungen zur Artenvielfalt und zur Untersuchung von ökosystemaren Fragestellungen. Das liegt auch daran, dass sich seit 1985 in der Bundesrepublik die höchste Spezialistendichte herausgebildet hat und die Sciaridenfauna Deutschlands im europäischen Maßstab am besten untersucht ist. Infolgedessen sind heute aus Deutschland meist doppelt so viele Arten bekannt, als aus jedem anderen Land Europas. Wegen der geringen Flugfähigkeit, der oft substratgebundenen Lebensweise und ihrer meist guten Anpassung an mikroklimatische Lebensräume sind die Trauermücken zur Interpretation einiger Biotoptypen einsetzbar. Zudem wird die phytozönotische Sukzession wesentlich von Besiedlungsprozessen der Sciaridae begleitet, da diese bereits kleinste Mikrokavernen als Lebensraum nutzen. Da nach gegenwärtigem Kenntnisstand ca. $80 \%$ aller Arten hygrophil sind, kann die Dipterenfamilie v. a. zur Beurteilung des Zustandes von Waldbiotopen, Mooren und Feuchtwiesen herangezogen werden. Gerade hier lassen sich wegen der höheren Beprobungsintensität standortspezifische Aussagen zur Attraktivität typischer Biotopstrukturen ableiten. In einigen Publikationen wurde schon darauf hingewiesen, dass die Trauermücken wahrscheinlich über ein hohes Potential bei der indikatorischen Widerspiegelung von bodenchemischen Kenngrößen verfügen. So vermuten bereits Rudzinski (1989b) und Schulz (1996) Zusammenhänge zwischen der Zusammensetzung von Sciaridenzönosen und dem pH-Wert des Waldbodens. Schulz (1996) stellt z. B. für 4 Laub- und Mischwaldflächen in Niederbayern heraus, dass an den Standorten mit den sauersten Böden auch relativ wenige Sciaridenarten schlüpfen. In bisher unveröffentlichten Projektberichten ist sogar davon die Rede, dass ausgewählte Spezies - wie z. B. Corynoptera vagula Tuомікоsкi - im Offenland sehr empfindlich auf Veränderungen des Grundwasserspiegels reagieren (Mohrig \& Heller 
pers. Mitt.). Offensichtlich gibt es einige stenöke Sciaridenspezies, die als Zeigerarten fungieren können. Auf dem intensiv genutzten Weidegrünland sowie auf Acker- und Ruderalflächen muss in Abhängigkeit von der angebauten Kultur, vom Düngegrad oder der Nutzungsintensität mit mehreren Ubiquisten gerechnet werden, die dann meist massenhaft auftreten (HeLler 1990, 2000; Rudzinski 1989a; Weber 1993; Weber \& Prescher 1995). Eine unkommentierte Übersicht dominanter Sciaridenarten, wie sie sich aus dem gegenwärtig verfügbaren Literaturfundus ergibt, wurde in Tabelle 2 zusammengestellt.

Als Untersuchungsgegenstand für bioindikatorische Studien eignet sich v. a. die Gruppe der phytosaprophagen Sciariden, zu der die sehr artenreichen Gattungen Epidapus, Bradysia, Corynoptera und Scatopsciara gerechnet werden. Zudem könnten auch Spezies aus anderen trophischen Gruppen - Phytophage [Cratyna, Phytosciara], Mycetophage [Lycoriella s. str.] und Karpo- bzw. Xylophage [Schwenckfeldina, Scythropochroa, Trichosia, Xylosciara] - eine Zeigerfunktion besitzen (Tabelle 2).

Die Sciaridae haben mehrere Eigenschaften, die sie als Zeigergruppe einerseits als besonders geeignet und andererseits als problembehaftet erscheinen lassen. Folglich müssen bei der Planung und Durchführung von ökologischen Studien folgende Gegebenheiten berücksichtigt werden:

\section{Vorteile}

1. Hohe Vermehrungsraten und schnelle Entwicklungszyklen führen unter günstigen klimatischen Verhältnissen zu hohen Siedlungsdichten und zu einem hohen Anteil an der Biomasseproduktion der Diptera (HövEMEYER 1984, 1985). Daraus ergibt sich eine stetig hohe Präsenz des Untersuchungsgegenstandes, so dass meist über den gesamten Jahresverlauf repräsentative Materialaufsammlungen möglich sind.

2. Die mitunter sehr kurze Generationsdauer und das daraus resultierende Vermehrungspotential befähigt die Trauermücken zu kurzfristigen Reaktionen auf günstige oder ungünstige Umweltbedingungen (Heller 1990). Den Vorteil von kleinen Organismen mit kurzen Lebenszyklen beim Monitoring diskutiert auch CAIRns (2002).

3. Mit Hilfe der Sciaridae lassen sich bodenchemische, sukzessionsbedingte und/oder durch anthropogene Nutzung verursachte Veränderungen in der Kulturlandschaft nachweisen (u. a. Heller 2000, 2002; Rudzinski 1989a, b; Mohrig 1967; Weber \& Prescher 1995; WERNER 1997).

4. Die Sciaridenimagines der meisten Spezies zeigen eine geringe Flugaktivität und besitzen eine oft substratgebundene Lebensweise. In manchen Gattungen [z. B. Epidapus und Corynoptera] kommen v. a. im weiblichen Geschlecht aptere oder brachyptere Formen vor. Daraus ergibt sich bei hinreichenden Lebensbedingungen eine gewisse „Standorttreue“ (MoHrig 1967).

5. Die Horizontalverteilung der Dipterenlarven richtet sich in Waldbiotopen offenbar nach der Zusammensetzung der Krautschicht aus (HövemeYer 1984). Außerdem haben die Zusammensetzung der Bodenstreu, deren Mächtigkeit, Alter und Beschattung, der pH-Wert des Bodens sowie das Feuchtigkeitsregime Einfluss auf die Sciaridenzönose. Bei Kenntnis dieser Parameter und der sich daraus ergebenden Zusammenhänge können vom Trauermücken-Spezialisten geeignete Probeflächen ausgewählt und das potentielle Arteninventar abgeschätzt werden.

6. Viele Sciaridenarten zeichnen sich durch eine hohe Nachweisstetigkeit aus. Bei den Freilandarbeiten besitzt jedoch die gruppenspezifisch abgestimmte Methodenwahl eine ergebnisbestimmende Bedeutung. Folglich müssen die Leerungszyklen, die einzusetzenden Fallentypen und deren Kombination auf die ökologische Fragestellung abgestimmt sein. 
7. Die Trauermücken können mit nahezu jeder Fangmethodik und - mit Ausnahme von Gewässern - in jedem Biotop erbeutet werden (Menzel \& Mohrig 2000).

8. Qualitative Aussagen zum Arteninventar sind bereits bei vertretbarem Zeitaufwand mittels Kescherstreifmethode erzielbar. Dabei sollten die Untersuchungsflächen mindestens ein- bis zweimal während der Vegetationsperioden [Frühjahrs-, Sommer- und Herbstaspekt] ganztägig aufgesucht werden. Bei abgetrockneter Vegetation und bei Mindesttemperaturen von $15^{\circ} \mathrm{C}$ haben sich die Tageszeiten zwischen 11.00 Uhr und 18.00 Uhr - im Hochsommer sogar von 10.00 Uhr bis 19.00 Uhr - als günstig erwiesen. Besonders gute Sammelergebnisse werden bei Sonnenschein und Windstille erreicht.

9. Beim Sammeln mit dem Netzkescher können die Sciaridenimagines aufgrund ihres auffälligen Verhaltens schnell erkannt und mit dem Exhaustor extrahiert werden. Im Gegensatz zu allen anderen Insekten zeichnen sich Trauermücken durch schnelle, zielgerichtete Laufbewegungen auf dem Gazenetz aus, wobei die Flügel in der Regel über dem Abdomen zusammengelegt sind. Kurze Flugbewegungen werden von den Imagines meist nur ausgeführt, wenn sie sich an der Netzwandung schnell aufrichten müssen. Aufgrund dieses Verhaltensmusters lassen sich selbst kleinste Imagines im Gelände erkennen und problemlos fangen.

10. Die Sciaridae-Imagines sind zumindest unter den Nematocera recht kompakt und robust gebaute Tiere. Dadurch werden sie - im Gegensatz zu anderen Mückengruppen [z. B. Cecidomyiidae, Limoniidae oder Tipulidae] - bei Sammel- und Sortierarbeiten kaum beschädigt.

11. Die vorsortierten Sciaridenproben lassen sich in $70 \%$-igem Alkohol [mit oder ohne Vergällungszusatz] problemlos über einen längeren Zeitraum lagern. Extreme Farbausbleichungen, die z. B. bei den Cecidomyiidae in kürzester Zeit zum Nichterkennen von wichtigen Körpermerkmalen und damit zur Unbestimmbarkeit führen können, kommen bei den Sciaridae zumindest in den ersten 10 Jahren der Alkoholkonservierung nicht vor.

12. Alle morphologischen Feinstrukturen [einschließlich der männlichen Genitalien], die für die Artbestimmung von Bedeutung sein können, liegen bei den Sciaridae frei und sind dem Determinator mit optischen Geräten zugänglich. Dadurch wird die Präparation und die Determination wesentlich erleichtert.

13. Für Aussagen zur Diversität einer Sciaridenzönose müssen in der Regel nur die Männchen und die flügelreduzierten Weibchen präpariert werden. Nach einer gewissen Übung ist die Zuordnung der Imagines, welche zu besonders häufigen Sciaridenspezies gehören, bereits unter dem Binokular möglich (HeLLer 1990).

\section{Nachteile}

1. Die Populationsdichte kann von Jahr zu Jahr sehr großen Schwankungen unterworfen sein (u. a. Hövemeyer 1984, 1992a).

2. Langzeituntersuchungen können aufgrund der zu erwartenden Materialmengen arbeitsund kostenintensiv werden. In Abhängigkeit vom Witterungsverlauf fallen mitunter extrem hohe Individuenzahlen an, die sich oft jedoch nur auf wenige Sciaridenarten verteilen. In Malaisefallen und Gelbschalen wurden schon bis zu 10.000 Exemplare je Fangprobe und Woche gezählt.

3. Bestimmungsarbeiten im Gelände sind nicht möglich. Die Materialproben sind stets in 70 \%-igem Alkohol aufzubewahren. Von einer Bearbeitung getrockneter Imagines wird abgeraten, weil sich dadurch die Merkmalserkennung erschwert und zusätzliche präparatorische Arbeitsschritte erforderlich werden [vergleiche hierzu Menzel \& MoHrig (2000)]. 
4. Die Präparation erfordert einen vergleichsweise hohen Zeit- und Materialaufwand, da zumindest von den kleinen und im Habitus sehr ähnlichen Formen Dauerpräparate herzustellen sind. Als Richtwert für den Präparationsumfang sollten in Abhängigkeit von der Probenzusammensetzung etwa 30 bis $50 \%$ der Männchen und $5 \%$ der Weibchen veranschlagt werden.

5. Bei kleinen Objekten mit ähnlichen Merkmalsstrukturen muss die Determination unter dem Mikroskop vorgenommen werden [Genitaluntersuchungen bei mindestens 400-facher Vergrößerung].

6. Eine schnelle Determination von seltenen bzw. sehr seltenen Sciaridenarten ist gegenwärtig nur möglich, wenn eine ausreichende Vergleichsammlung vorhanden ist. Die Sciaridae-Weibchen lassen sich bei fehlenden Männchen in den Alkohol-Proben nur schwer bestimmen.

\section{Zusammenfassung und Ausblick}

Die obigen Erläuterungen zeigen, dass wir heute ein viel breiteres Wissen über die Trauermücken im gesamtdeutschen Raum haben und über einen höheren Kenntnisstand bei der Beurteilung erhobener Daten verfügen, als noch vor 15 Jahren. Die Sciariden weisen Potentiale auf, um als Indikatorgruppe für Lebensraumeigenschaften und -qualitäten dienen zu können. Vermutlich sind manche Trauermückenarten auch als Bioindikatoren für bodenchemische Kennwerte einsetzbar. Ihr Potential für naturschutzfachliche Gutachten ist zunächst einmal auf Biodiversitätseinschätzungen beschränkt. Dass ökologische Freilandstudien für die Biodiversitätsforschung, den Arten- und Biotopschutz und darüber hinaus für die Systematik der Trauermücken bedeutsam sein können, wurde schon mehrfach herausgestellt (Menzel \& Mohrig 1991, Rudzinski 1993, Menzel et al. 2003, Menzel 2006, Menzel \& Heller 2006).

Eine besondere Verantwortung bei der Bewahrung von Lebensräumen in der Bundesrepublik Deutschland liegt vor, wenn Sciaridenspezies über die Landesgrenzen hinaus sehr selten sind oder wenn von einer Lokalität nur das Typenmaterial aus der Originalbeschreibung bekannt ist. Als Bewertungskriterium bei der Beurteilung des Seltenheitsgrades haben wir v. a. das Vorhandensein von sehr wenigen Fundmeldungen in Deutschland [maximal 5 Einzelnachweise] und das Fehlen von weiteren Nachweisen in Europa herangezogen. Eine computergestützte Analyse hat ergeben, dass zu den Kategorien „selten“ oder „sehr selten“ 146 Trauermückenarten gehören, was ca. $35 \%$ des in Deutschland verzeichneten Arteninventars ausmacht (Heller \& Menzel in prep. unter Einschluss unpublizierter Daten). Darunter befinden sich auch 37 Spezies, die zur Zeit aus keinem anderen europäischen Land bekannt sind. 26 Arten - von denen der locus typicus meist in Deutschland liegt - waren sogar nur an 1 oder 2 Lokalitäten nachweisbar. Darauf aufbauend zeichnet sich ab, dass zur Einschätzung der Gefährdungssituation und zur Ermittlung von Gefährdungsursachen auch aufbereitetes Datenmaterial herangezogen werden muss, das über Deutschland hinaus zusammengetragen wurde. Andererseits liegen dem Spezialisten nur wenige Informationen zur Entwicklungsbiologie der Trauermücken sowie über auftretende Populationsschwankungen und ihre Ursachen vor. Folglich sind fundierte Vorschläge für eine Rote Liste der Sciariden Deutschlands nur in der vorstehend besprochenen Komplexität möglich. Mit allgemeingültigen Seltenheitseinschätzungen (Menzel et al. 1990, Heller \& Menzel in prep.) und Beiträgen zur Roten Liste der Trauermücken in Brandenburg (Menzel 1992, Westendorff et. al. 1993, Sommer et al. 1994, TAeger et al. 1995) wurden die ersten Schritte zur Entwicklung wertgebender Indikationssysteme eingeleitet. 
Erfahrungsgemäß etablieren sich Indikatorgruppen erst dann, wenn die taxonomischen Grundlagen gefestigt sind und kompilatorische Bestimmungsschlüssel vorliegen. Diese Voraussetzungen sind bei den Trauermücken inzwischen erfüllt oder werden in naher Zukunft geschaffen. Denn mit der „Revision der paläarktischen Sciaridae“ (Menzel \& Mohrig 2000) liegt auch dem Nichtspezialisten ein Handbuch vor, das für nomenklatorische Sicherheit bei der Artbenennung sorgt und ein wichtiges Hilfsmittel beim Literaturstudium ist [v. a. Erkennen von synonymen Namen und artlichen Fehlbestimmungen]. Einige nomenklatorische Korrekturen zu diesem Basiswerk, die für den mitteleuropäischen Raum von Bedeutung sind, wurden von Hippa et al. (2003), Menzel \& Heller (2005, 2006), Menzel et al. (2006) und VilkamaA et al. (2004) vorgenommen. Auf der Basis einer neu erarbeiteten Systematik können die zu verifizierenden Arten den eingeschlossenen Gattungen, Untergattungen und Artengruppen sicher zugeordnet werden. Dadurch reduziert sich bei Bestimmungsarbeiten der Kreis der in Frage kommenden Arten beträchtlich. Obwohl die Nomenklatur und Systematik bei Tuomikoski (1960) nicht mehr den modernen Anforderungen entspricht, ist heute sein Beitrag "Zur Kenntnis der Sciariden Finnlands" für die Artdetermination noch unverzichtbar. Darauf aufbauend entstanden in den zurückliegenden Jahren mehrere Revisionen von Gattungen und Artengruppen, die wichtige Bestimmungstabellen, Redeskriptionen und gut figurierte Genitalstrukturen für die eingeschlossenen Spezies enthalten. Dazu gehören v. a. Bradysia s. l. [partim] (Mohrig \& Menzel 1993), Camptochaeta (Hippa \& VilkamaA 1994), Corynoptera s. l. [partim] (Hippa \& VilkamaA 1994; Mohrig 1993; Rulik et al. 2001), Dichopygina (Vilkamaa et al. 2004), Leptosciarella (Mohrig \& Menzel 1997), Phytosciara s. l. (Hippa \& Vilkamaa 1991, Mohrig \& Menzel 1994; Vilkamaa 2000), Trichodapus (Mohrig \& Menzel 1997), Trichosia (Menzel \& Mohrig 1997) und Xylosciara (Hippa \& Vilkamaa 2004). Handlungsbedarf besteht hingegen in mehreren Artengruppen von Bradysia s. l. und Corynoptera s. l. sowie in den Gattungen Epidapus, Lycoriella, Pseudolycoriella und Scatopsciara. In diesen Gruppen ist seit 1990 der höchste Artenzuwachs zu verzeichnen, was eine grundlegende Überarbeitung bestehender Bestimmungstabellen erfordert. Eine weitere Verbesserung der Situation wird eintreten, wenn 2008 das umfangreich bebilderte Handbuch zur Identifikation der Britischen Sciaridae (Menzel et al. in prep.) erscheint, mit dem auch die meisten mitteleuropäischen Arten bestimmbar sind.

In Bezug auf eine Instrumentalisierung von Trauermücken für ökologische Gutachten müsste man künftig versuchen, die vorgestellte Übersicht zu den zönologischen Koinzidenzen (Tabelle 2) weiter auszubauen und zu ergänzen. Ein Ziel könnte sein, daraus Charakterarten abzuleiten und euryöke Spezies auszuschließen. Im Ergebnis dessen könnte man eine Matrix für die Beurteilung von Lebensräumen anhand der Trauermücken erhalten. Das erfordert jedoch dringend weitere Studien, in denen die artspezifischen Habitatansprüche untersucht, Verbreitungsmuster herausgestellt und Gefährdungsstufen bzw. -ursachen abgeleitet werden.

\section{Danksagung}

Bei vielen Autoren, die im Text genannt werden, bedanken wir uns für das Bereitstellen von Literatur. Unser Dank gilt auch den Herren Kai Heller (Heikendorf) und Prof. Dr. Werner Mohrig (Poseritz) für zahlreiche Diskussionen, das Bereitstellen von Daten und eine kritische Durchsicht des Manuskriptes. Die Fotografien stellten uns freundlicherweise Frank Köhler (Bornheim) [Fig. 1] und Christian Stierstorfer (Moos) [Fig. 2-3] zur Verfügung.

\section{Literatur}

AltmülLER, R. 1977: Ökoenergetische Untersuchungen an Dipterenpopulationen im Buchenwald. [133-138]. - Verhandlungen der Gesellschaft für Ökologie 1976. 6. Jahresversammlung vom 20. bis 24. September 1976 in Göttingen: v-xv und 1-622; The Hague [Den Haag]: Dr. W. Junk bv Publishers. 
AltmüLleR, R. 1979: Untersuchungen über den Energieumsatz von Dipterenpopulationen im Buchenwald (Luzulo-Fagetum). - Pedobiologia 19 (4): 245-278; Jena.

Anderson, J. M. 1975: The enigma of soil animal species diversity. [51-58]. - In: VAneK, J. (Hrsg.): Progress in soil zoology. Proceedings of the 5th International Colloquium on Soil Zoology held in Prague September 17-22, 1973: 1-407; Prague: Academia, Publishing House of the Czechoslovak Academy of Sciences.

Bogenschütz, H. 2005: Über die Arthropodenfauna des „Bechtaler Waldes“. - WSG Baden-Württemberg 8: 125-168; Freiburg.

Borrmann, K. 2004: Heereswurm - Wanderzug in den Heiligen Hallen. - Labus. Naturschutz im Land Mecklenburg-Strelitz 20; Neustrelitz.

Brauns, A. 1991: Taschenbuch der Waldinsekten: 1-860; Stuttgart: Gustav-Fischer-Verlag.

BUNR [= Bundesministerium für Umwelt, Naturschutz und Reaktorsicherheit] 2002: Bericht der Bundesregierung nach dem Übereinkommen über die biologische Vielfalt - Nationalbericht biologische Vielfalt: 1-152; Bonn: Neusser-Verlag.

CAIRns, J. 2002: Environmental monitoring for the preservation of global biodiversity: the role in sustainable use of the planet. - International Journal of Sustainable Development and World Ecology 9 (2): 135-150; Carnforth.

Dorn, K. 1987: Dipterenemergenzen in PCP-belasteten Waldböden des Staatswaldes Burgholz - die Nematoceren im Buchen- und Fichtenforst, Teil I. - Jahresberichte des Naturwissenschaftlichen Vereins in Wuppertal 40: 77-89; Wuppertal.

DrEws, U. 1986: Ökofaunistische Untersuchungen der Sciaridenfauna des Mecklenburger Landrückens. - Diplomarbeit, Ernst-Moritz-Arndt-Universität Greifswald, Sektion Biologie, Wissenschaftsbereich Zoologie: 1-68; Greifswald [unveröffentlicht].

Dunger, W. 1983: Tiere im Boden. - Die neue Brehm-Bücherei 327: 1-280; Wittenberg Lutherstadt: A. Ziemsen-Verlag.

Eckert, R.; Mohrig, W. \& Kallweit, U. 1999: Ein Beitrag zur Mückenfauna (Trauer- und Pilzmücken) der Höhlen deutscher Mittelgebirge (Harz, Kyffhäuser, Thüringer Wald, Zittauer Gebirge). - Mitteilungen des Verbandes deutscher Höhlen- und Karstforscher 45 (2): 66-70; München.

Ellenberg, H.; Mayer, R. \& Schauermann, J. 1986: Ökosystemforschung-Ergebnisse des Sollingprojekts 1966-1986: 1-508; Stuttgart: Ulmer-Verlag.

Elmer, M. \& Roth, M. 2001: Effekte des Umbaus von Fichtenmonokulturen in Buchenmischwälder auf die saprophagen Dipteren. - Mitteilungen der Deutschen Gesellschaft für allgemeine und angewandte Entomologie 13 (1-6): 319-324; Giessen.

FALINSKI, J. B. 1978: Uprooted trees, their distribution and influence in the primeval forest biotope. Vegetatio 38: 175-183; Dordrecht.

Feldmann, R. 1991: Die Makrofauna auf Kiefern- und Buchenstandorten im Mainzer Kalkflugsandgebiet. Artenspektrum der Dipteren, Biomasse und Konsumtion der Makrosaprophagen, Auswirkungen von Kalkgaben. - Dissertation, Johann-Gutenberg-Universität Mainz: 1-178; Mainz.

Feldmann, R. 1992: Die Bodenmakrofauna im Lennebergwald. 1. Die Dipteren. - Mainzer Naturwissenschaftliches Archiv 30: 171-241; Mainz.

Fourman, K. L. 1938: Untersuchungen über die Bedeutung der Bodenfauna bei der biologischen Umwandlung des Bestandesabfalles forstlicher Standorte. - Mitteilungen aus Forstwirtschaft und Forstwissenschaft, 1938 (2): 144-169; Hannover.

Fritz, H.-G. 1982: Ökologische und systematische Untersuchungen an Diptera / Nematocera (Insecta) in Überschwemmungsgebieten des nördlichen Oberrheins. Ein Beitrag zur Ökologie großer Flußauen. - Dissertation, Technische Hochschule Darmstadt, Fachbereich 10 - Biologie: 1-296; Darmstadt.

Fritz, H.-G. 1983a: Wenig bekannte und neue Diptera/Nematocera (Insecta) aus der nördlichen Oberrheinaue. - Andrias 3: 9-24; Karlsruhe. 
FriTz, H.-G. 1983b: Strukturanalyse der Diptera/Nematocera (Mücken) in ephemeren Lebensräumen des nördlichen Oberrheingebietes. - Verhandlungen der Gesellschaft für Ökologie (Mainz 1981) 10: 307-311; Göttingen.

Froese, A. 1992: Vergleichende Untersuchungen zur Biologie und Ökologie der Dipteren auf integriert und konventionell bewirtschafteten Feldern. - Dissertation, Justus-Liebig-Universität Gießen, Institut für Phytopathologie und Angewandte Zoologie / Eberhard-Karls-Universität Tübingen, Lehrstuhl für Spezielle Zoologie: 1-248; Gießen.

Frouz, J. 1999: Use of soil dwelling Diptera (Insecta, Diptera) as bioindicators: a review of ecological requirements and response to disturbance. - Agriculture, Ecosystems and Environment 74: 167-186; Amsterdam.

FunKe, W. 1971: Food and energy turnover of leaf-eating insects and their influence on primary production. [81-93]. - In: Ellenberg, H. (Hrsg.): Integrated experimental ecology. - Ecological Studies 2: 1-214; Berlin, Heidelberg \& New York: Springer-Verlag.

Haslett, J. R. 2001: Biodiversity and Conservation of Diptera in heterogeneous land mosaics: A fly's eye view. - Journal of Insect Conservation 5 (2): 71-75: London.

Heller, K. 1990: Vergleichend ökologische Untersuchungen an terricol-detritophagen Nematocera zur biozönotischen Struktur und zum Stoff- und Energiefluß in einem Wald-Agrar-Ökosystemkomplex. - Diplomarbeit, Christian-Albrechts-Universität zu Kiel, Zoologisches Institut: 1-143; Kiel [unveröffentlicht].

Heller, K. 1996: Vergleichende biozönotische und populationsbiologische Untersuchungen an terricol-detriophagen Nematocera in einem Wald-Agrar-Ökosystemkomplex. [41-85]. - In: HeYdEmann, B.; Hofmann, W. \& Irmler, U. (Hrsg.): Funktionen und Interaktionen der Fauna in einer Wald-Agrar-Landschaft Schleswig-Holsteins. - Faunistisch-Ökologische Mitteilungen (Supplement) 22: 1-126; Kiel.

Heller, K. 1998: Beiträge zur Sciaridenfauna Schleswig-Holsteins (Diptera). Teil 1. Das Trentmoor bei Plön. - Dipteron 1 (3): 45-56; Kiel.

Heller, K. 1999: Trauermücken (Diptera) von Gönnersdorf (Kr. Daun). Beiträge zur Insektenfauna der Eifeldörfer XX. - Dendrocopos 26: 249-262; Konz.

HelLer, K. 2000: Naturschutzmanagement im Feuchtgrünland und seine Auswirkungen auf bodenlebende Nematocera (Diptera: Sciaridae). - Mitteilungen der Deutschen Gesellschaft für allgemeine und angewandte Entomologie 12 (1-6): 611-614; Giessen.

Heller, K. 2002a: Beiträge zur Sciaridenfauna Schleswig-Holsteins (Diptera, Sciaridae). Teil 3. Die Sciariden des Projektgebietes „Integrierter Landbau Rade“. - Dipteron 4 (2): 109-124; Kiel.

Heller, K. 2002b: Nachträge zur Sciaridenfauna Brandenburgs (Diptera: Sciaridae). [Kurzmitteilung Nr. 3/2002 - Faunistik]. - Studia dipterologica 9 (1): 189-190; Halle (Saale).

HelLER, K. \& Büchs, W. 2003: Nachtrag zur Sciaridenfauna Sachsen-Anhalts (Diptera, Sciaridae). - Studia dipterologica 10 (1): 179-180; Halle (Saale).

Heller, K. \& Menzel, F. (in prep.): Ergänzungen zur Trauermücken-Fauna Deutschlands, nebst der Beschreibung von 9 neuen Arten (Diptera: Sciaridae). - Beiträge zur Entomologie [in Vorbereitung]; Keltern.

Hennicke, S.; Martschei, T. \& Müller-Motzfeld, G. 1997: Erste Ergebnisse der Erfassung ausgewählter Arthropodengruppen der Stadt Greifswald. - Insecta 5: 51-100; Berlin.

Hippa, H. \& VilkamaA, P. 1991: The genus Prosciara Frey (Diptera, Sciaridae). - Entomologica Fennica 2 (3): 113-155; Helsinki.

Hippa, H. \& VilkamaA, P. 1994: The genus Camptochaeta gen. nov. (Diptera, Sciaridae). - Acta Zoologica Fennica 194: 1-85; Helsinki.

Hippa, H. \& VilkamaA, P. 2004: The genus Xylosciara Tuomikoski (Diptera, Sciaridae): phylogeny and review of the species. - Acta Zoologica Fennica 214: 1-38; Helsinki. 
Hippa, H.; VilkamaA, P. \& Mohrig, W. 2003: Phylogeny of Corynoptera Winnertz and related genera, with the description of Claustropyga gen. nov. (Diptera, Sciaridae). - Studia dipterologica 9 (2) (2002): 469-511; Halle (Saale).

Holstein, J. 1990: Sciaridenpopulationen (Diptera, Nematocera) eines Fichtenforstes. Zönologische und populationsökologische Untersuchungen. - Diplomarbeit, Mathematisch-naturwissenschaftliche Fakultät der Universität Ulm: 1-86; Ulm [unveröffentlicht].

Holstein, J. \& Funke, W. 1993: Die Sciaridenzönose eines Fichtenforstes (Diptera: Nematocera). Mitteilungen der Deutschen Gesellschaft für allgemeine und angewandte Entomologie 8 (4-6): 641-647; Giessen.

Hövemeyer, K. 1984: Die Dipterengemeinschaft eines Buchenwaldes auf Kalkgestein: Produktion an Imagines, Abundanz und räumliche Verteilung insbesondere der Larven. - Pedobiologia 26: 1-15; Jena.

HövemeYer, K. 1985: Die Zweiflügler (Diptera) eines Kalkbuchenwaldes: Lebenszyklen, Raum-Zeit-Muster und Nahrungsbiologie. - Dissertation, Georg-August-Universität zu Göttingen: 1-280; Göttingen.

Hövemeyer, K. 1987: Reaktionen von Dipteren auf experimentell veränderten Laubstreufall in einem Buchenwald. - Jahresberichte des Naturwissenschaftlichen Vereins in Wuppertal 40: 5-9; Wuppertal.

HövemeYer, K. 1989a: Bradysia drakenbergensis, spec. nov., eine neue Sciaridenart aus dem südniedersächsischen Bergland (BRD). (Diptera, Sciaridae). - Spixiana 12 (2): 209-211; München.

Hövemeyer, K. 1989b: Der Einfluß von Streumenge und Streuqualität auf die Siedlungsdichte von Dipterenlarven: ein Freilandexperiment im Kalkbuchenwald (Zur Funktion der Fauna in einem Mullbuchenwald 4). - Verhandlungen der Gesellschaft für Ökologie (Göttingen 1987) 17: 229-236; Göttingen.

Hövemeyer, K. 1989c: Die Dipterengemeinschaft einer extensiv genutzten Mähwiese. - DGaaE Nachrichten 3 (3): 59-60; Darmstadt.

Hövemeyer, K. 1991: The study of dipterous populations and communities in European terrestrial ecosystems. [99-109]. - In: Weismann, L.; Országh, I. \& Pont, A. C. (Hrsg.): Proceedings of the Second International Congress of Dipterology, Bratislava, August 27 - September 1, 1990: 1-367; Bratislava: VEDA, Publishing House of the Slovak Academy of Sciences \& The Hague [Den Haag]: SPB Academic Publishing.

Hövemeyer, K. 1992a: Die Dipterengemeinschaft eines Kalkbuchenwaldes: eine siebenjährige Untersuchung. - Zoologische Jahrbücher, Abteilung für Systematik, Ökologie und Geographie der Tiere 119 (2): 225-260; Jena.

Hövemeyer, K. 1992b: Response of Diptera populations to experimentally modified leaf litter input in a beech forest on limestone. - Pedobiologia 36 (1): 35-49; Jena.

Hövemeyer, K. 1996a: Die Dipterengemeinschaft eines Erlenuferwaldes in Südniedersachsen. - Braunschweiger naturkundliche Schriften 5 (1): 71-84; Braunschweig.

Hövemeyer, K. 1996b: Die Dipterengemeinschaften eines Halbtrockenrasens und einer Hecke im südniedersächsischen Bergland: eine vergleichende Untersuchung. - Drosera 1996 (2): 113-127; Oldenburg.

Hövemeyer, K. 1997: Die Dipterengemeinschaft eines südniedersächsischen Eichen-Hainbuchenwaldes. - Göttinger naturkundliche Schriften 4: 137-150; Göttingen.

Hövemeyer, K. 1998: Diptera associated with dead beech wood. - Studia dipterologica 5 (1): 113-122; Halle (Saale).

Hövemeyer, K. 1999: Abundance patterns in terrestrial dipteran communities. - Pedobiologia 43 (1): 28-43; Jena.

Hövemeyer, K. \& Schauermann, J. 2003: Succession of Diptera on dead beech wood: a 10-year study. - Pedobiologia 47: 61-75; Jena.

Irmler, U.; Heller, K. \& Warning, J. 1996: Age and tree species as factors influencing the populations of insects living in dead wood (Coleoptera, Diptera: Sciaridae, Mycetophilidae). - Pedobiologia 40 (2): 134-148; Jena.

Kratochwil, A. \& Schwabe, A. 2001: Ökologie der Lebensgemeinschaften: Biozönologie: 1-756; Stuttgart: Ulmer-Verlag. 
Kühne, S.; Schrameyer, K.; Müller, R. \& Menzel, F. 1994: Räuberische Fliegen - ein bisher wenig beachteter Nützlingskomplex in Gewächshäusern. - Mitteilungen aus der Biologischen Bundesanstalt für Land- und Forstwirtschaft Berlin-Dahlem 302: 3-75; Berlin.

LaSAlle, J. \& Gauld, I. D. 1993: Hymenoptera and biodiversity: 1-348; Wallingford: CAB International.

Lavelle, P.; Bignell, D.; Lepage, M. 1997: Soil function in a changing world: the role of invertebrate ecosystem engineers. - European Journal of Soil Biology 33 (4): 159-193; Paris.

Lembкe, K. 1986: Ökologisch-faunistische Untersuchungen der Sciaridenfauna des Mecklenburger Landrückens. - Diplomarbeit, Ernst-Moritz-Arndt-Universität Greifswald, Sektion Biologie, Wissenschaftsbereich Zoologie: 1-47 [+ 8 Seiten Anhang]; Greifswald [unveröffentlicht].

LeuCKefeld, S. 1995: Untersuchungen zur Sciaridenfauna der innerstädtischen Elster-Pleiße-Aue von Leipzig (Insecta: Diptera: Sciaridae). - Wissenschaftliche Hausarbeit zur Erlangung des 1. Staatsexamens für das Lehramt am Gymnasium, Universität Leipzig, Fachbereich Biowissenschaften, Abteilung Zoologie: 1-91; Leipzig [unveröffentlicht].

Markel, M. 2002a: Trauermücke überraschte Wanderer auf ihrem Weg zum Brocken. - Der Harz 2002 (1): 20; Wernigerode.

Markel, M. 2002b: Heerwürmer - Unheilsverkünder? - Der Harz 2002 (1): 20; Wernigerode.

Menzel, F. 1992: Trauermücken (Sciaridae). [141-142]. - In: Ministerium für Umwelt, Naturschutz und Raumordnung des Landes Brandenburg (Hrsg.): Rote Liste. Gefährdete Tiere im Land Brandenburg: 1-288; Potsdam: Unze Verlagsgesellschaft mbh.

Menzel, F. 2000: Die Trauermücken-Fauna der Bundesrepublik Deutschland (Diptera: Sciaridae). Beiträge zur Entomologie 50 (2): 317-355; Berlin.

Menzel, F. 2006: 6.13.7 Familie Trauermücken (Sciaridae). [204-208]. - In: Nationalparkverwaltung Berchtesgaden (Hrsg.): Quellen im Nationalpark Berchtesgaden. Lebensgemeinschaften als Indikatoren des Klimawandels. - Nationalpark Berchtesgaden, Forschungsbericht 51: 1-272; Berchtesgaden.

Menzel, F. \& Heller, K. 2005: Sechs neue Arten aus den Gattungen Bradysia, Camptochaeta und Corynoptera (Diptera: Sciaridae) nebst einigen Bemerkungen zur Nomenklatur europäischer Trauermücken. - Studia dipterologica 11 (2) (2004): 335-357; Halle (Saale).

Menzel, F. \& Heller, K. 2006: Trauermücken (Diptera: Sciaridae) aus dem Nationalpark „Hainich“ (Thüringen) nebst der Beschreibung von Scatopsciara andrei Menzel spec. nov. - Studia dipterologica 13 (1): 41-55; Halle (Saale).

Menzel, F; Heller, K. \& SMith, J. 2002: Neue Trauermücken-Nachweise (Diptera: Sciaridae) aus dem Harz nebst der Beschreibung einer neuen Bradysia-Art. - Studia dipterologica 9 (1): 179-189; Halle (Saale).

Menzel, F. \& Mohrig, W. 1991: Beiträge zur Faunistik und Ökologie des Naturschutzgebietes „Apfelstädter Ried“, Kreis Erfurt-Land. Teil VI - Diptera: Sciaridae. - Veröffentlichungen des Naturkundemuseums Erfurt 10: 27-45; Erfurt.

Menzel, F. \& Mohrig, W. 1997: Revision der paläarktischen Arten von Trichosia Winnertz sensu Tuomikoski, 1960 (Diptera, Sciaridae). - Teil I. Gattung Trichosia Winnertz, 1867. - Studia dipterologica 4 (1): 3-40; Halle (Saale).

Menzel, F. \& Mohrig, W. 1998: Beiträge zur Taxonomie und Faunistik der paläarktischen Trauermücken (Diptera, Sciaridae). Teil VI - Neue Ergebnisse aus Typenuntersuchungen und die daraus resultierenden taxonomisch-nomenklatorischen Konsequenzen. - Studia dipterologica 5 (2): 351-378; Halle (Saale).

Menzel, F. \& Mohrig, W. 2000: Revision der paläarktischen Trauermücken (Diptera: Sciaridae). - Studia dipterologica Supplement 6 (1999): 1-761; Halle (Saale).

Menzel, F.; Mohrig, W. \& Groth, I. 1990: Beiträge zur Insektenfauna der DDR: Diptera - Sciaridae. - Beiträge zur Entomologie 40 (2): 301-400; Berlin.

Menzel, F; Schulz, U. \& Taeger, T. 2003: Neue Trauermücken-Funde aus dem nordostdeutschen Tiefland, mit einer ökologischen Betrachtung von Wurzelteller-Fängen und einer Checkliste der aus Berlin/Brandenburg bekannten Arten (Diptera: Sciaridae). - Beiträge zur Entomologie 53 (1): 71-105; Keltern. 
Menzel, F.; Smith, J. E. \& Chandler, P. J. 2006: The sciarid fauna of the British Isles (Diptera: Sciaridae), including descriptions of six new species. - Zoological Journal of the Linnean Society 146 (1): 1-147; London.

Menzel, F.; Smith, J. E. \& Chandler, P. J. (in prep.): Dark Winged Fungus Gnats. Diptera, Sciaridae. - Handbooks for the Identification of British Insects: [in Vorbereitung]; London.

Messner, B.; Mohrig, W.; Moritz, M. \& Broen, B. von 1982: Ergebnisse zoologischer Untersuchungen in Großhöhlen des Harzes und des Kyffhäusers. [Anlage zum Festkolloquium 50 Jahre organisierte Höhlenforschung im Harz, 17.-19. November 1978, Bad Frankenhausen - Teil II]. - Die Grotte 5 (1): 1-8; Erfurt.

MetZner, K. 1993: Untersuchungen zur Sciaridenfauna des innerstädtischen Auwaldgebietes Burgaue bei Leipzig (Insecta: Diptera: Sciaridae). - Wissenschaftliche Hausarbeit zur Erlangung des 1. Staatsexamens für das Lehramt am Gymnasium, Universität Leipzig, Fachbereich Biowissenschaften, Abteilung Zoologie: 1-69; Leipzig [unveröffentlicht].

Metzner, K. \& MenZel, F. 1996: Untersuchungen zur Sciaridenfauna des innerstädtischen Auwaldgebietes Burgaue bei Leipzig (Insecta, Diptera, Sciaridae). - Studia dipterologica 3 (1): 125-154; Halle (Saale).

Moнrig, W. 1967: Beitrag zur Ökologie und Verbreitung brachypterer Dipteren in norddeutschen Biotopen. - Deutsche Entomologische Zeitschrift (Neue Folge) 14 (1-2): 169-184; Berlin.

Mohrig, W. 1993: Der Artenkreis Corynoptera concinna (Winnertz 1867) (Diptera, Sciaridae). - Bonner Zoologische Beiträge 44 (1-2): 47-55; Bonn.

Mohrig, W.; Broen, B. von; Messner, B. \& Moritz, M. 1968: Beiträge zur Arthropodenfauna aus Großhöhlen des Harzes und des Kyffhäusers. I. Allgemeine Charakteristik der untersuchten Höhlen und Fundortbeschreibung. II. Diptera. - Deutsche Entomologische Zeitschrift (Neue Folge) 15 (4-5): 367-387; Berlin.

Mohrig, W. \& Hövemeyer, K. 1992: Vier neue Trauermückenarten aus Südniedersachsen (BRD). (Diptera, Sciaridae). - Spixiana 15 (3): 269-273; München.

Mohrig, W. \& Menzel, F. 1993: Revision der paläarktischen Arten der Bradysia brunnipes-Gruppe (Diptera, Sciaridae). - Bonner Zoologische Beiträge 44 (3-4): 267-291; Bonn.

Mohrig, W. \& Menzel, F. 1994: Revision der paläarktischen Arten von Phytosciara Frey (Diptera: Sciaridae). - Beiträge zur Entomologie 44 (1): 167-210; Berlin.

Mohrig, W. \& Menzel, F. 1997: Revision der paläarktischen Arten von Trichosia Winnertz sensu Tuомікоsкі, 1960 (Diptera, Sciaridae). - Teil II. Gattungen Leptosciarella Tuомікоsкі, 1960 und Trichodapus gen. nov. - Studia dipterologica 4 (1): 41-98; Halle (Saale).

Paquin, P. \& Coderre, D. 1997: Changes in soil macroarthropod communities in relation to forest maturation through succesional stages in Canadian boreal forests. - Oecologia 112: 104-111; Berlin \& Heidelberg.

Plachter, H. 1991: Naturschutz: 1- 463; Stuttgart: Gustav-Fischer-Verlag.

Rотн, M. 2005: Ein Wandererlebnis aus Steinach im Thüringer Wald - Beobachtungen zum Heerwurm (Diptera: Sciaridae). - Mitteilungen des Thüringer Entomologenverbandes e.V. 12 (1): 13-15; Gotha.

RudZINSKI, H.-G. 1989a: Zur Schlüpfabundanz von Trauermücken auf unterschiedlichen Flächen einer abgedeckten Bauschuttdeponie (Diptera: Sciaridae). - Mitteilungen des Internationalen Entomologischen Vereins e.V. 14 (1-2): 27-38; Frankfurt am Main.

Rudzinski, H.-G. 1989b: Der Einfluß von Schadstoffbelastung (Bodenversauerung) und Düngungsmaßnahmen auf die Abundanzdynamik der Trauermücken in Fichtenbeständen des Fichtelgebirges (Diptera, Nematocera: Sciaridae). - Nachrichtenblatt der bayerischen Entomologen 38 (3): 71-78; München.

Rudzinski, H.-G. 1992a:Zum Vorkommen von Pilz- und Trauermücken in unterschiedlichen Fichtenforsten Nordostbayerns (Diptera, Nematocera: Mycetophilidae, Sciaridae). - Entomofauna 13 (26): 425-442; Ansfelden. 
Rudzinski, H.-G. 1992b: Beiträge zur Kenntnis der Trauermückenfauna Nordwestdeutschlands (Diptera, Nematocera: Sciaridae). - Drosera 1992 (1): 35-45; Oldenburg.

Rudzinski, H.-G. 1993: Mücken und Fliegen aus dem Schluifelder Moos, Ober-Bayern. Zweite Liste (Diptera: Nematocera: Sciaridae). - Entomofauna 14 (16): 281-304; Ansfelden.

Rudzinski, H.-G. 1994: Zum Vorkommen von Dipteren auf einem Hausbalkon. Teil II: Sciaridae, Hybotidae und Empididae. - Entomologische Zeitschrift 104 (23): 461-468; Essen.

Rulik, B.; Mohrig, W. \& Jaschhof, M. 2001: Trauermücken (Diptera: Sciaridae) und freilebende Gallmücken (Diptera: Cecidomyiidae: Lestremiinae) aus Ungarn. Mit Bemerkungen zur Corynoptera tridentata-Gruppe. - Folia Entomologica Hungarica 62: 231-245; Budapest.

SACHER, P. 2001: Wandern am Brocken - einmal ganz anders. - Der Harz 2001 (11): 12-13; Wernigerode.

SCHRANKel, I. 1998: Faunistisch-ökologische Charakterisierung ausgewählter Quellen im Nationalpark Berchtesgaden. - Diplomarbeit, Universität des Saarlandes, Fachrichtung Biogeographie: 1-79 [+ 26 Seiten Anhang]; Saarbrücken [unveröffentlicht].

Schulz, U. 1996: Vorkommen und Habitatanforderungen von Bodenmakroarthropoden in Natur- und Wirtschaftswäldern: ein Vergleich. - Dissertation, Forstliche Fakultät der Ludwig-Maximilians-Universität München: 1-166; München.

Schulz, U. 2001: Biologische Vielfalt in Wäldern und forstlicher Einfluss. [143-150]. - In: Bundesamt für Naturschutz (Hrsg.): Treffpunkt Biologische Vielfalt - Aktuelle Forschung im Rahmen des Übereinkommens über die biologische Vielfalt vorgestellt auf einer wissenschaftlichen Expertentagung an der Internationalen Naturschutzakademie Insel Vilm vom 4.-8. September 2000: 1-247; Münster-Hiltrup: Landwirtschaftsverlag GmbH.

Schulz, U. 2002: Betrachtung der Zoodiversität in Wäldern - Bedeutung von Skalen, Strukturen und Substraten. - Beiträge für Forstwirtschaft und Landschaftsökologie 36 (2): 49-54; Berlin.

Sommer, M.; Taeger, A.; Westendorff, M. \& Ziegler, J. 1994: Arthropodenarten der Roten Liste Brandenburgs im Biosphärenreservat Schorfheide-Chorin. - Brandenburger Entomologische Nachrichten 2 (1): 63-77; Potsdam.

Sous-Dorn, B. \& Dorn, K. 1990: Die Dipteren aus immissionsbelasteten Waldökosystemen in Berlin (West) unter besonderer Berücksichtigung der Tanzfliegen (Empididae) und Trauermücken (Sciaridae). - Zoologische Beiträge (Neue Folge) 33 (2): 197-207; Berlin.

Taeger, A.; Behne, L.; Blank, S.; Broen, B. von; Burger, F.; Dathe, H. H.; Gaedike, R.; Groll, E.; Menzel, F.; Pellmann, H.; Sommer, M.; Westendorff, M.; Zerche, L. \& Ziegler, J. 1995: Untersuchungen der Arthropodenfauna im Biosphärenreservat Schorfheide-Chorin in den Jahren 1992 bis 1994. - Projektbericht in 2 Bänden (Allgemeiner Teil \& Faunistischer Teil), Deutsches Entomologisches Institut: 1-313; Eberswalde [unveröffentlicht].

TAEger, T. 2000: Naturschutzfachliche und entomofaunistische Bedeutung von aufgeklappten Wurzeltellern in brandenburgischen Wäldern. Vergleichende Untersuchungen an verschiedenen Wurzelteller-Typen der Buche. - Diplomarbeit, Fachhochschule Eberswalde, Fachbereich Landschaftsnutzung und Naturschutz: 1-101; Eberswalde [unveröffentlicht].

Thiede, U. 1977: Untersuchungen über die Arthropodenfauna in Fichtenforsten (Populationsökologie, Energieumsatz). - Zoologische Jahrbücher, Abteilung für Systematik, Ökologie und Geographie der Tiere 104: 137-202; Jena.

Tuомікоsкi, R. 1960: Zur Kenntnis der Sciariden (Diptera) Finnlands. - Annales Zoologici Societatis Zoologicae Botanicae Fennicae „Vanamo“ 21 (4): 1-164; Helsinki.

VilkamaA, P. 2000: Phylogeny of Prosciara Frey and related genera (Diptera: Sciaridae). - Systematic Entomology 25 (1): 47-72; Oxford.

Vilkamaa, P.; Hippa, H. \& Komarova, L. A. 2004: The genus Dichopygina gen. n. (Diptera: Sciaridae). - Insect Systematics and Evolution 35 (1): 107-120; Copenhagen. 
WARNING, J. 1991: Freilandökologische Untersuchungen zur Besiedlung von Totholz durch Insecta in verschiedenen Waldökosystemen in Abhängigkeit von Art, Alterszustand und Struktur des Holzes. - Diplomarbeit, Christian-Albrechts-Universität zu Kiel, Zoologisches Institut: 1-106; Kiel [unveröffentlicht].

Weber, D. 1991: Die Evertebratenfauna der Höhlen und künstlichen Hohlräume des Katastergebietes Westfalen einschließlich der Quellen- und Grundwasserfauna. - Abhandlungen zur Karst- und Höhlenkunde 25: 1-701; München.

Weber, D. 2001: Die Höhlenfauna und -flora des Höhlenkatastergebietes Rheinland-Pfalz/Saarland. 4. Teil. - Abhandlungen zur Karst- und Höhlenkunde 33: 1-322; München.

Weber, G. 1993: Die Nematocera (Insecta: Diptera) eines klärschlammgedüngten und schwermetallbelasteten Ackers: Ökologie und Larvalbiologie. - Dissertation, Naturwissenschaftliche Fakultät der Technischen Universität Carolo-Wilhelmina zu Braunschweig: 1-162; Braunschweig.

WeBER, G. \& BüCHs, W. 1995: Der Einfluß eines unterschiedlich intensiven Einsatzes von landwirtschaftlichen Produktionsmitteln auf die Mückenfauna (Diptera: Nematocera) in der Kultur Zuckerrübe. - Mitteilungen der Deutschen Gesellschaft für allgemeine und angewandte Entomologie 9 (4-6): 635-640; Giessen.

Weber, G. \& Büchs, W. 1997: Einfluß eines langjährig unterschiedlich intensiven Einsatzes von Pflanzenschutz- und Düngemitteln auf die Schlüpfabundanzen ausgewählter Familien der Nematocera (Diptera) einer Zuckerrübenfruchtfolge. - Verhandlungen der Gesellschaft für Ökologie 27: 373-377; Stuttgart, Jena, Lübeck \& Ulm.

Weber, G.; Franzen, J. \& Büchs, W. 1995: Dipteren als Zersetzer von toter organischer Substanz in Agrarökosystemen. - Mitteilungen der Deutschen Gesellschaft für allgemeine und angewandte Entomologie 10 (1-6): 491-495; Giessen.

Weber, G.; Franzen, J. \& Büchs, W. 1997: Beneficial Diptera in field crops with different inputs of pesticides and fertilizers. - Biological Agriculture and Horticulture 15: 109-122; Coventry.

Weber, G. \& Prescher, S. 1995: Die Mücken und Fliegen eines klärschlammgedüngten Ackers. Agrarökologie 15: 5-100; Bern/Stuttgart/Wien.

Weidemann, G. \& Schauermann, J. 1986: Die Tierwelt, ihre Nahrungsbeziehungen und ihre Rolle. [179 266]. - In: Ellenberg, H. \& Mayer, R. \& Schauermann, J. (Hrsg.): Ökosystemforschung - Ergebnisse des Solling-Projekts: 1-507; Berlin, Heidelberg \& New York: Verlag Eugen Ulmer.

Werner, D. 1997: Die Dipterenfauna verschiedener Mülldeponien und Kompostierungsanlagen in der Umgebung von Berlin unter besonderer Berücksichtigung ihrer Ökologie und Bionomie. - Studia dipterologica Supplement 1: 1-176; Halle (Saale).

Westendorff, M.; Taeger, A. \& Sommer, M. 1993: Erste Ergebnisse von Untersuchungen der Arthropodenfauna im Biosphärenreservat Schorfheide-Chorin. - Brandenburger Entomologische Nachrichten 1 (1): 53-56; Potsdam.

Wolters, V. 1998: Functional aspects of animal diversity in soil - introduction and overview. - Applied Soil Ecology 10: 185-190; Amsterdam.

Zachariae, G. 1965: Spuren tierischer Tätigkeit im Boden des Buchenwaldes. - Forstwissenschaftliche Forschungen (Beiheft Forstwissenschaftliches Centralblatt) 20: 1-68; Berlin, Hamburg \& Heidelberg: Parey-Verlag.

\section{Anschriften der Verfasser:}

Dr. Frank Menzel

Deutsches Entomologisches Institut

Leibniz-Zentrum für Agrarlandschafts-

forschung (ZALF)

Eberswalder Straße 84

D - 15374 Müncheberg, Germany
Prof. Dr. UlRich Schulz

Fachhochschule Eberswalde

Fachbereich Landschaftsnutzung

und Naturschutz

Friedrich-Ebert-Straße 28

D - 16225 Eberswalde, Germany

\section{Subject editor:}

Prof. Dr. K. Hövemmeyer 


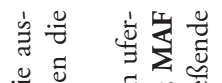

$\because \frac{\square}{\square}$

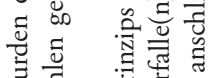

峝 茄

ปี

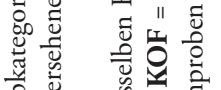

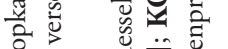

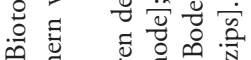

ปี हี

प्ष

घ छ

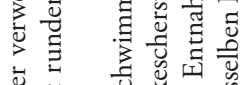

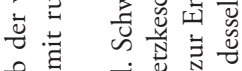

寻

可

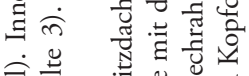

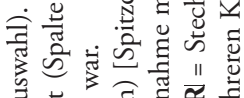

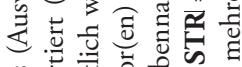

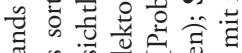

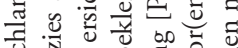

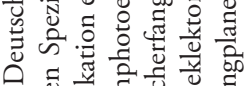

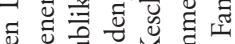
o.

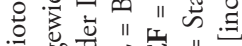
论

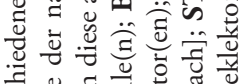

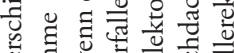
है है $\exists$ के 풘 矛 氜密完

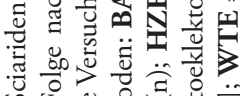

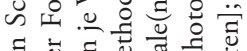

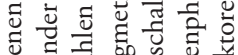
.

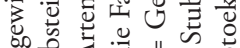

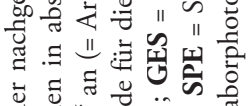

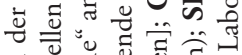

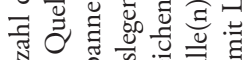
定专 की

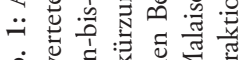

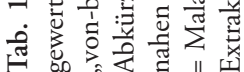

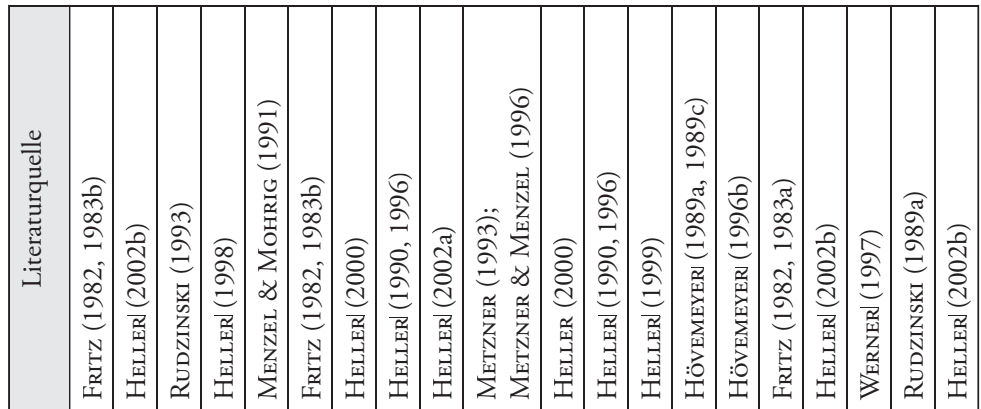

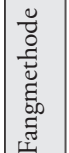

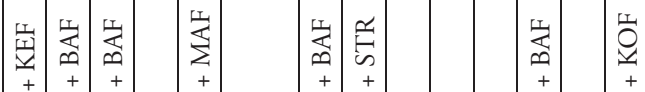

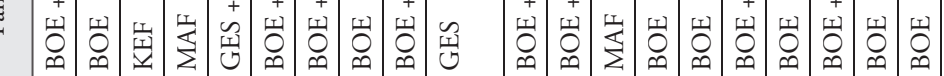

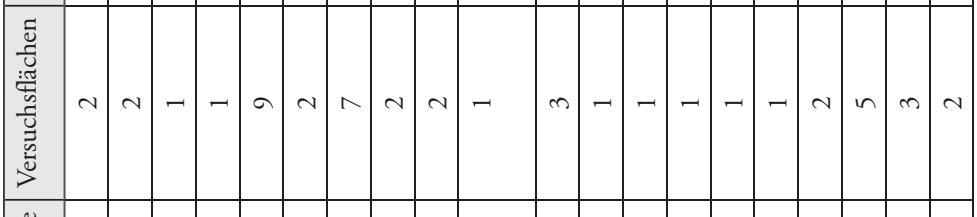

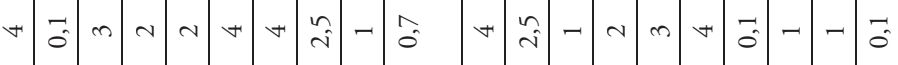

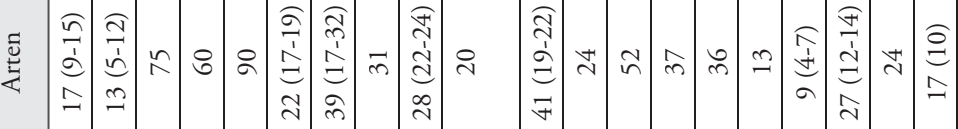

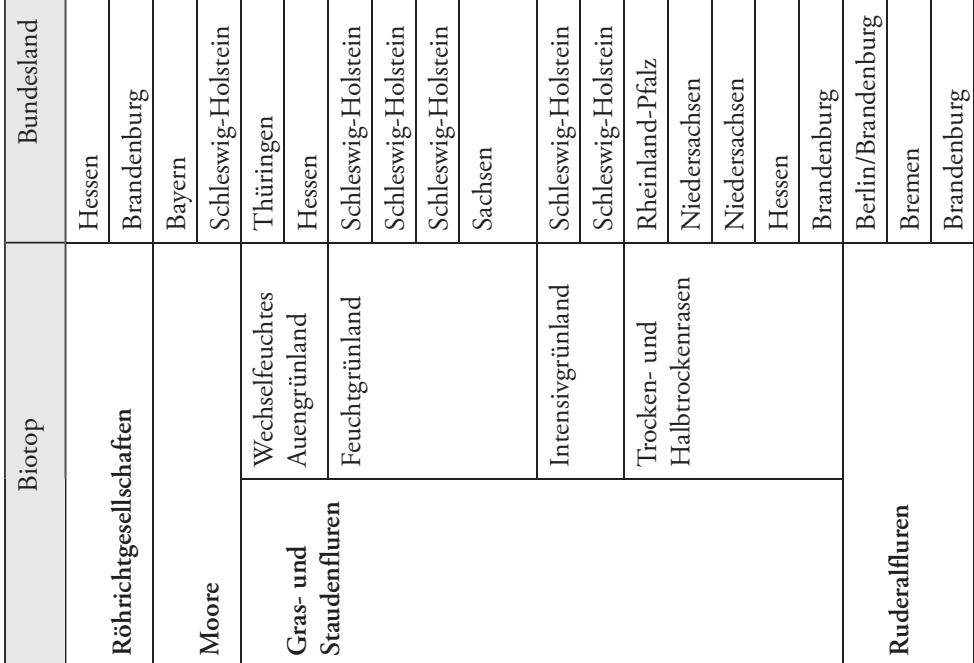




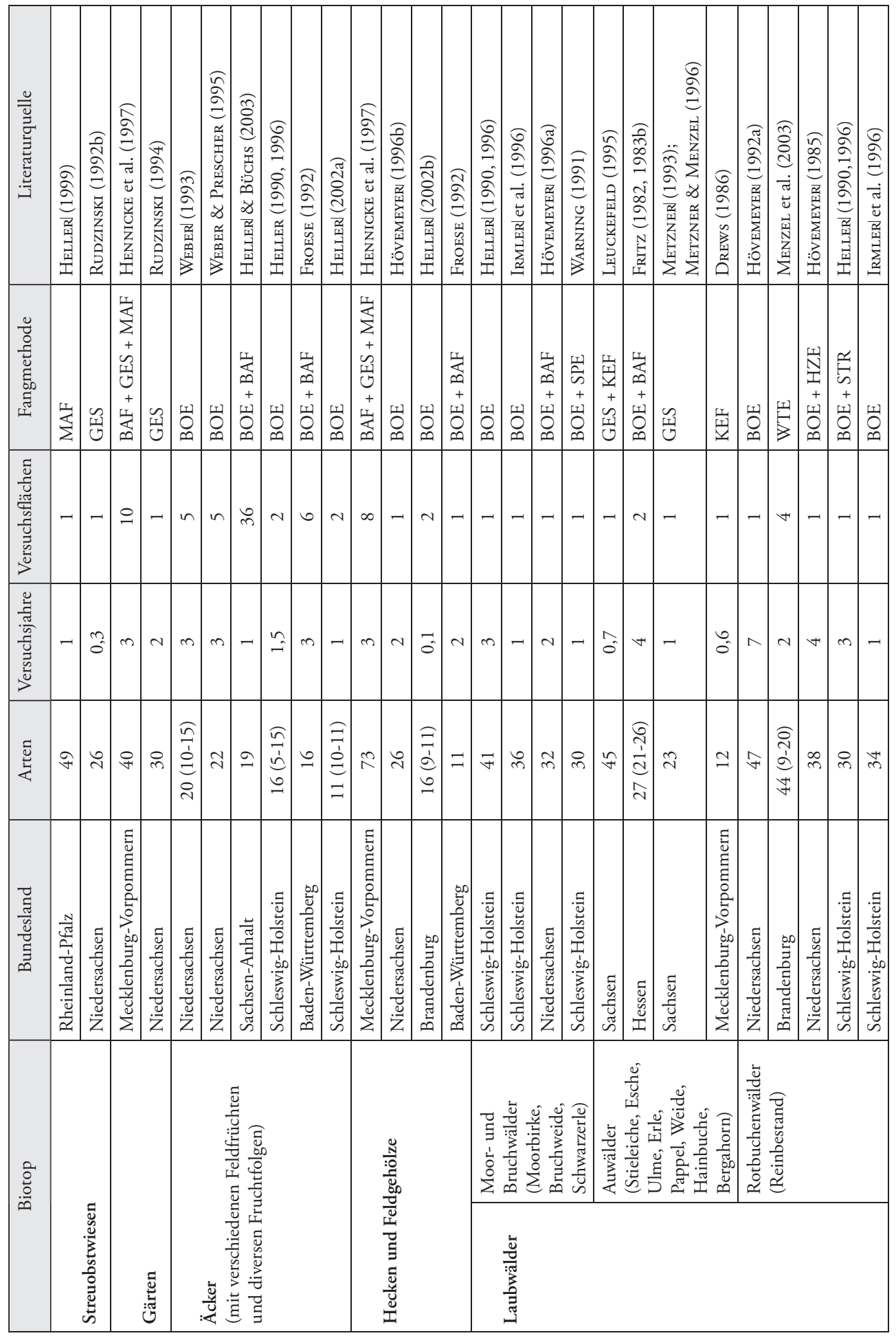




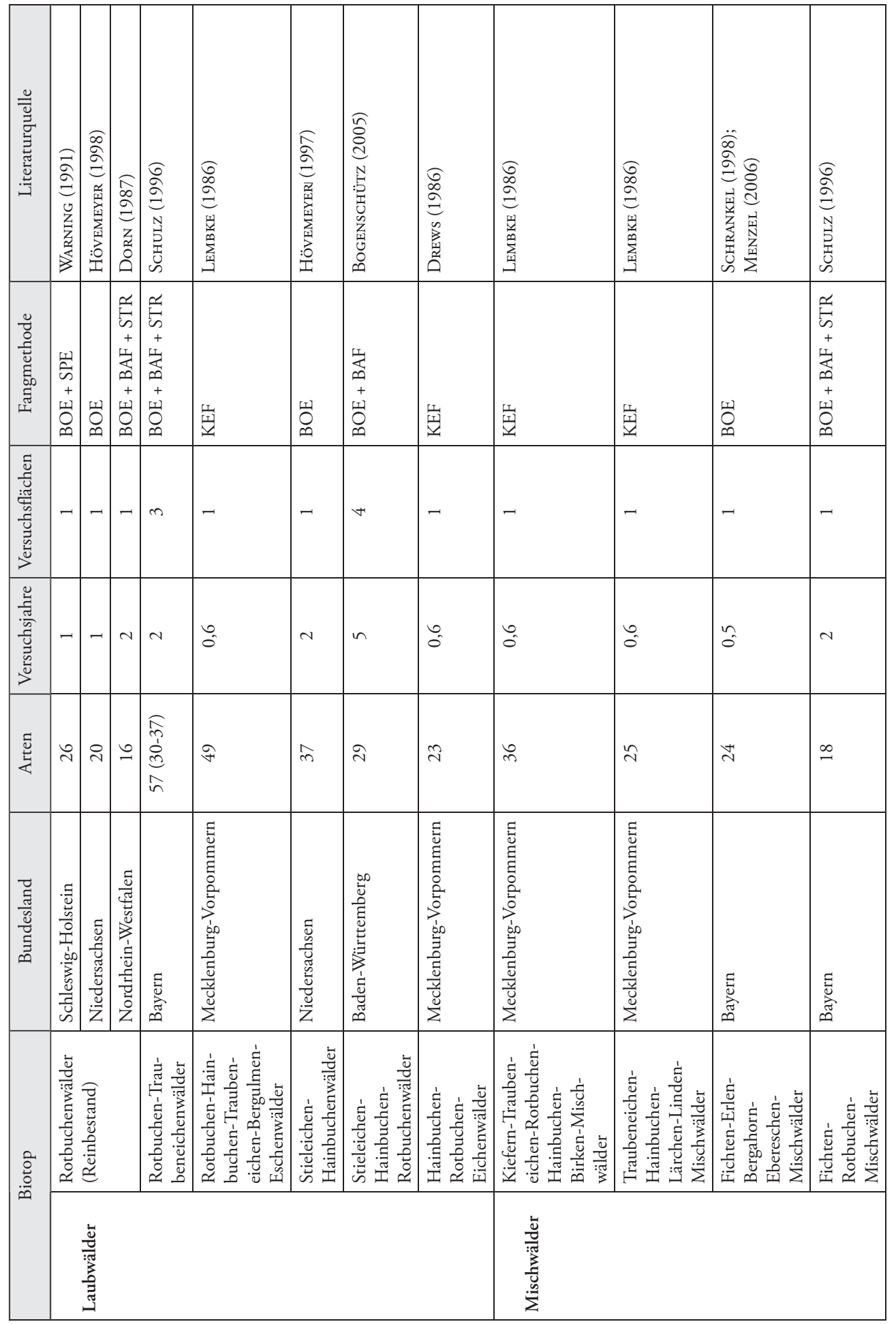




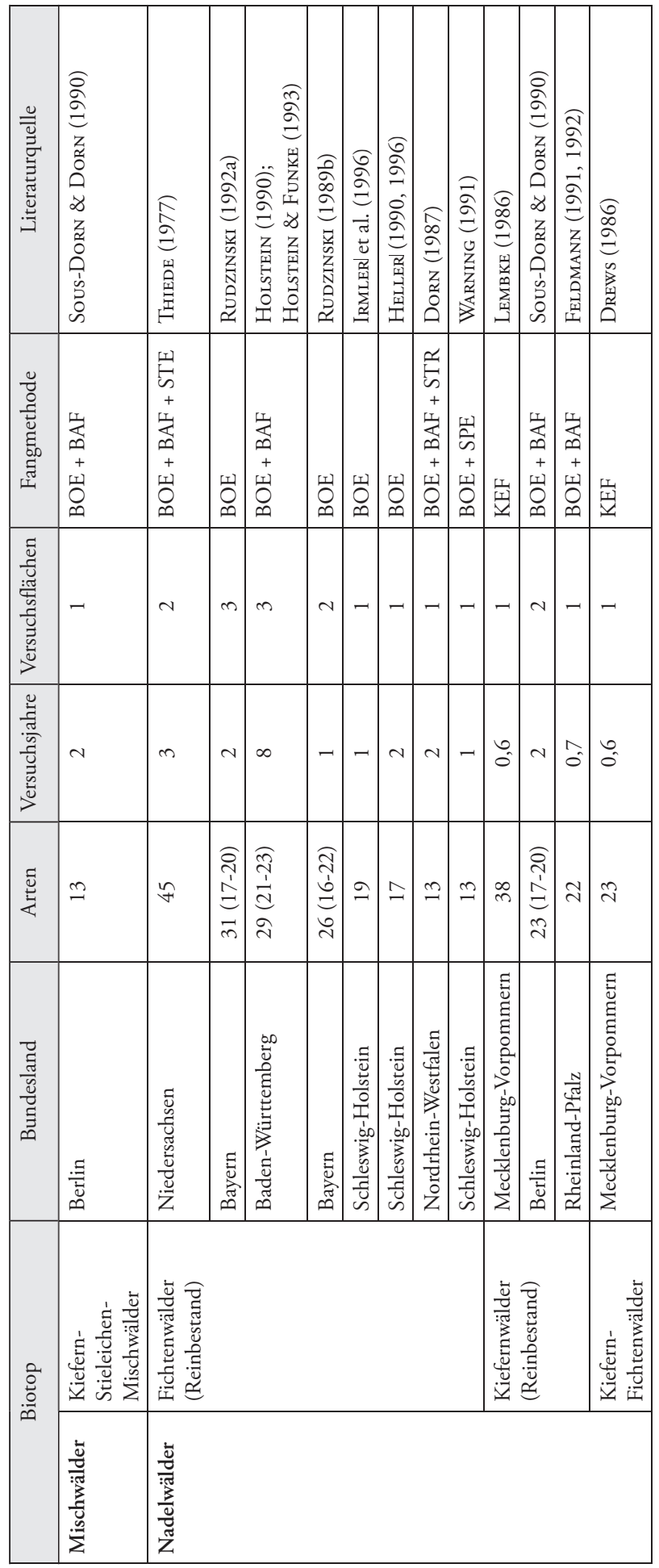


Tab. 2: Zönologische Koinzidenzen (dominante Sciaridenarten mit hoher Nachweisstetigkeit) - eine unkommentierte Zusammenstellung aus der Literatur. Die verwendete Nomenklatur und Systematik richtet sich nach Menzel \& Mohrig (2000) und Menzel \& Heller (2005, 2006). Überprüfte Fehlbestimmungen oder artliche Fehldeutungen wurden kommentarlos berichtigt (vergl. hierzu mit den Literaturzitationen in Menzel \& Mohrig 2000).

\begin{tabular}{|c|c|c|c|}
\hline \multicolumn{2}{|c|}{ Biotop } & dominante Sciaridae-Arten & Literaturquellen \\
\hline \multicolumn{2}{|c|}{ Röhrichtgesellschaften } & $\begin{array}{l}\text { Bradysia nitidicollis (MEIGEN, 1818) } \\
\text { Corynoptera fera MOHRIG \& B HeLLER, } 1992 \\
\text { Corynoptera perpusilla WINNERTZ, 1867 } \\
\text { Corynoptera tetrachaeta TuOMIKOSKI, 1960 } \\
\text { Lycoriella castanescens (LENGERSDORF, 1940) } \\
\text { Lycoriella conspicua (WINNERTZ, 1867) } \\
\text { Scatopsciara simillima (TuOMIKOSKI, 1960) }\end{array}$ & $\begin{array}{l}\text { Fritz (1982, 1983b); } \\
\text { HeLLer (2002b) }\end{array}$ \\
\hline \multicolumn{2}{|l|}{ Moore } & \begin{tabular}{|l|} 
Bradysia kassebeeri HELLER, 1998 \\
Bradysia nitidicollis (MEIGEN, 1818) \\
Bradysia praecox (MEIGEN, 1818) \\
Bradysiopsis vittigera (ZETTERSTEDT, 1851) \\
Cratyna colei (FREEMAN, 1990) \\
Cratyna falcifera (LENGERSDORF, 1933) \\
Cratyna nobilis (WINNERTZ, 1867) \\
Cratyna spiculosa (RUDZINSKI, 1993) \\
Phytosciara saetosa (LENGERSDORF, 1929) \\
Pseudolycoriella japonensis (MOHRIG \& MENZEL, 1992) \\
Scatopsciara multispina (BUKOWSKI \& \\
LENGERSDORF, 1936) \\
Scatopsciara vitripennis (MEIGEN, 1818) \\
Sciara humeralis ZETTERSTEDT, 1855
\end{tabular} & $\begin{array}{l}\text { HELLER (1998); } \\
\text { MOHRIG \& MENZEL } \\
\text { (1994); } \\
\text { RUDZINSKI (1993) }\end{array}$ \\
\hline \multirow[t]{2}{*}{$\begin{array}{l}\text { Gras- und } \\
\text { Staudenfluren }\end{array}$} & $\begin{array}{l}\text { Wechselfeuchtes } \\
\text { Auengrünland }\end{array}$ & $\begin{array}{l}\text { Bradysia nitidicollis (MEIGEN, 1818) } \\
\text { Bradysia scabricornis TUOMIKOSKI, 1960 } \\
\text { Bradysia polonica (LENGERSDORF, 1929) } \\
\text { Corynoptera membranigera (KIEFFER, 1903) } \\
\text { Corynoptera perpusilla WINNERTZ, 1867 } \\
\text { Corynoptera saccata TUOMIKOSKI, 1960 } \\
\text { Corynoptera tetrachaeta TUOMIKOSKI, 1960 } \\
\text { Lycoriella castanescens (LENGERSDORF, 1940) } \\
\text { Scatopsciara atomaria (ZETTERSTEDT, 1851) } \\
\text { Scatopsciara multispina (BUKOWSKI \& } \\
\quad \text { LENGERSDORF, 1936) } \\
\text { Scatopsciara simillima (TUOMIKOSKI, 1960) } \\
\text { Scatopsciara vitripennis (MEIGEN, 1818) }\end{array}$ & $\begin{array}{l}\text { Fritz (1982, 1983b); } \\
\text { MenZel \& Mohrig } \\
\text { (1991) }\end{array}$ \\
\hline & Feuchtgrünland & $\begin{array}{l}\text { Bradysia brevispina TUOMIKOSKI, 1960 } \\
\text { Bradysia inusitata TUOMIKOSKI, 1960 } \\
\text { Bradysia nitidicollis (MEIGEN, 1818) } \\
\text { Bradysia polonica (LENGERSDORF, 1929) } \\
\text { Bradysia scabricornis TUOMIKOSKI, 1960 } \\
\text { Bradysia subscabricornis MOHRIG \& MENZEL, } 1990 \\
\text { Cratyna colei (FreEMAN, 1990) } \\
\text { Cratyna falcifera (LENGERSDORF, 1933) } \\
\text { Cratyna nobilis (WINNERTZ, 1867) } \\
\text { Lycoriella castanescens (LENGERSDORF, 1940) } \\
\text { Sciara humeralis ZETTERSTEDT, 1851 } \\
\end{array}$ & $\begin{array}{l}\text { Heller (1990, 1996, } \\
\text { 2000, 2002a); } \\
\text { MENZEL et al. (1990) }\end{array}$ \\
\hline
\end{tabular}




\begin{tabular}{|c|c|c|c|}
\hline \multicolumn{2}{|c|}{ Biotop } & dominante Sciaridae-Arten & Literaturquellen \\
\hline \multirow[t]{2}{*}{$\begin{array}{l}\text { Gras- und } \\
\text { Staudenfluren }\end{array}$} & Intensivgrünland & $\begin{array}{l}\text { Bradysia flavipila TUOMIKOSKI, } 1960 \\
\text { Bradysia nitidicollis (MEIGEN, 1818) } \\
\text { Bradysia scabricornis TUOMIKOSKI, 1960 } \\
\text { Bradysia trivittata (STAEGER, 1840) } \\
\text { Corynoptera tetrachaeta TUOMIKOSKI, } 1960 \\
\text { Lycoriella castanescens (LENGERSDORF, 1940) } \\
\text { Scatopsciara atomaria (ZETTERSTEDT, 1851) }\end{array}$ & $\begin{array}{l}\text { HELLER }(1990,1996, \\
\text { 2000) }\end{array}$ \\
\hline & $\begin{array}{l}\text { Trocken- und } \\
\text { Halbtrocken- } \\
\text { rasen }\end{array}$ & $\begin{array}{l}\text { Bradysia drakenbergensis HÖVEMEYER, } 1989 \\
\text { Bradysia trivittata (STAEGER, 1840) } \\
\text { Corynoptera bistrispina (BUKOWSKI \& } \\
\quad \text { LENGERSDORF, 1936) } \\
\text { Corynoptera flavicauda (ZETTERSTEDT, 1855) } \\
\text { Corynoptera hypopygialis (LENGERSDORF, 1926) } \\
\text { Corynoptera luteofusca (BUKOWSKI \& } \\
\quad \text { LENGERSDORF, 1936) } \\
\text { Corynoptera perpusilla WINNERTZ, } 1867 \\
\text { Corynoptera saetistyla MOHRIG \& KRIVOSHEINA, } 1985 \\
\text { Corynoptera subparvula TUOMIKOSKI, } 1960 \\
\text { Corynoptera tridentata HONDRU, 1968 } \\
\text { Cratyna falcata (TUOMIKOSKI, 1960) } \\
\text { Cratyna vagabunda (WINNERTZ, 1867) } \\
\text { Epidapus montivivus (MOHRIG, 1970) } \\
\text { Parapnyxia latifurcata (LENGERSDORF, 1942) } \\
\end{array}$ & $\begin{array}{l}\text { FRITZ (1982, 1983a, } \\
\text { 1983b); } \\
\text { HELLER (1999, 2002b); } \\
\text { HÖVEMEYER (1989a, } \\
\text { 1989c, 1996b); } \\
\text { MENZEL et al. (1990, } \\
\text { 2002); } \\
\text { MOHRIG \& HöVEMEYER } \\
\text { (1992); } \\
\text { TAEGER et al. (1995) }\end{array}$ \\
\hline \multicolumn{2}{|l|}{ Ruderalfluren } & $\begin{array}{l}\text { Bradysia nitidicollis (MEIGEN, 1818) } \\
\text { Bradysia trivittata (STAEGER, 1840) } \\
\text { Corynoptera perpusilla WINNERTZ, 1867 } \\
\text { Lycoriella castanescens (LENGERSDORF, 1940) } \\
\text { Scatopsciara atomaria (ZETTERSTEDT, 1851) } \\
\text { Sciara hemerobioides (SCOPOLI, 1763) }\end{array}$ & $\begin{array}{l}\text { HELLER (2002b); } \\
\text { RUdZINSKI (1989a); } \\
\text { WERNER (1997) }\end{array}$ \\
\hline \multicolumn{2}{|l|}{ Streuobstwiesen } & $\begin{array}{l}\text { Bradysia scabricornis TUOMIKOSKI, } 1960 \\
\text { Bradysia trivittata (STAEGER, 1840) } \\
\text { Bradysia vagans (WINNERTZ, 1868) } \\
\text { Corynoptera tridentata HONDRU, } 1968 \\
\text { Cratyna vagabunda (WINNERTZ, 1867) }\end{array}$ & $\begin{array}{l}\text { HELLER (1999); } \\
\text { RudZINSKI (1992b) }\end{array}$ \\
\hline \multicolumn{2}{|l|}{ Gärten } & $\begin{array}{l}\text { Bradysia difformis FREY, } 1948 \\
\text { Bradysia nitidicollis (MEIGEN, 1818) } \\
\text { Bradysia tilicola (LOEW, 1850) } \\
\text { Scatopsciara atomaria (ZETTERSTEDT, 1851) } \\
\end{array}$ & RuDZINSKI (1994) \\
\hline \multicolumn{2}{|c|}{$\begin{array}{l}\text { Äcker } \\
\text { (mit verschiedenen Feldfrüchten } \\
\text { und diversen Fruchtfolgen) }\end{array}$} & $\begin{array}{l}\text { Bradysia angustipennis WINNERTZ, } 1867 \\
\text { Bradysia angustoocularis MOHRIG \& KRIVOSHEINA, } 1989 \\
\text { Bradysia forcipulata (LUNDBECK, 1898) } \\
\text { Bradysia strenua (WINNERTZ, 1867) } \\
\text { Bradysia trivittata (STAEGER, 1840) } \\
\text { Bradysia urticae MOHRIG \& MENZEL, } 1992 \\
\text { Bradysia vagans (WINNERTZ, 1868) } \\
\text { Corynoptera cruciata (HIPPA \& VILKAMAA, 1994) } \\
\text { Corynoptera perpusilla WINNERTZ, 1867 } \\
\text { Lycoriella castanescens (LENGERDORF, 1940) } \\
\text { Lycoriella secundaria MOHRIG \& MENZEL, 1990 } \\
\text { Pseudolycoriella subbruckii (MOHRIG \& } \\
\quad \text { HövEMEYER, 1992) } \\
\text { Scatopsciara atomaria (ZETTERSTEDT, 1851) } \\
\text { Scatopsciara vitripennis (MEIGEN, 1818) } \\
\text { Hyperlasion wasmanni SCHMITZ, 1919) }\end{array}$ & 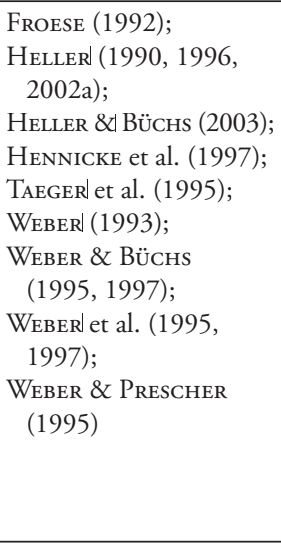 \\
\hline
\end{tabular}




\begin{tabular}{|c|c|c|c|}
\hline \multicolumn{2}{|c|}{ Biotop } & dominante Sciaridae-Arten & Literaturquellen \\
\hline \multicolumn{2}{|c|}{ Hecken und Feldgehölze } & $\begin{array}{l}\text { Bradysia nitidicollis (MEIGEN, 1818) } \\
\text { Bradysia regularis (LENGERSDORF, 1934) } \\
\text { Bradysia urticae MOHRIG \& MENZEL, } 1992 \\
\text { Bradysia vagans (WINNERTZ, 1868) } \\
\text { Corynoptera fera MOHRIG \& HELLER, 1992 } \\
\text { Corynoptera flavicauda (ZETTERSTEDT, 1855) } \\
\text { Corynoptera perpusilla WINNERTZ, 1867 } \\
\text { Corynoptera subparvula TUOMIKOSKI, } 1960 \\
\text { Corynoptera tridentata HONDRU, 1968 } \\
\text { Epidapus absconditus (VIMMER, 1926) } \\
\text { Epidapus ignavus (LENGERSDORF, 1941) } \\
\text { Lycoriella castanescens (LENGERSDORF, 1940) } \\
\text { Scatopsciara atomaria (ZETTERSTEDT, 1851) }\end{array}$ & $\begin{array}{l}\text { Froese (1992); } \\
\text { HeLler (2002b); } \\
\text { HöveMEyer (1996b) }\end{array}$ \\
\hline \multirow[t]{3}{*}{ Laubwälder } & $\begin{array}{l}\text { Moor- und } \\
\text { Bruchwälder } \\
\text { (Moorbirke, } \\
\text { Bruchweide, } \\
\text { Schwarzerle) }\end{array}$ & $\begin{array}{l}\text { Bradysia angustipennis WINNERTZ, } 1867 \\
\text { Bradysia aprica (WINNERTZ, 1867) } \\
\text { Bradysia fungicola (WINNERTZ, 1867) } \\
\text { Bradysia inusitata TUOMIKOSKI, 1960 } \\
\text { Bradysia scabricornis TUOMIKOSKI, } 1960 \\
\text { Corynoptera subdentata MOHRIG, } 1985 \\
\text { Corynoptera cursor (HIPPA \& VILKAMAA, 1994) } \\
\text { Corynoptera trepida (WINNERTZ, 1867) } \\
\text { Epidapus absconditus (VIMMER, 1926) } \\
\text { Epidapus alnicola (TUOMIKOSKI, 1957) } \\
\text { Epidapus gracilis (WALKER, 1848) } \\
\text { Scatopsciara atomaria (ZETTERSTEDT, 1851) } \\
\text { Trichosia morio (FABRICIUS, 1794) }\end{array}$ & $\begin{array}{l}\text { HELLER }(1990,1996) ; \\
\text { HöVEMEYER (1996a); } \\
\text { IRMLER et al. (1996); } \\
\text { MOHRIG (1967); } \\
\text { WARNING (1991) }\end{array}$ \\
\hline & $\begin{array}{l}\text { Auwälder } \\
\text { (Stieleiche, } \\
\text { Esche, } \\
\text { Ulme, Erle, } \\
\text { Pappel, Weide, } \\
\text { Hainbuche, } \\
\text { Bergahorn) } \\
\end{array}$ & $\begin{array}{l}\text { Bradysia scabricornis TUOMIKOSKI, } 1960 \\
\text { Corynoptera membranigera (KIEFFER, 1903) } \\
\text { Corynoptera saccata TUOMIKOSKI, 1960 } \\
\text { Epidapus atomarius (DE GEER, 1778) } \\
\text { Epidapus microthorax (BöRNER, 1903) } \\
\text { Schwenckfeldina carbonaria (MEIGEN, 1830) } \\
\text { Zygoneura sciarina MEIGEN, } 1830 \\
\end{array}$ & $\begin{array}{l}\text { Drews (1986); } \\
\text { Fritz(1982); } \\
\text { LEUCKEFELD (1995); } \\
\text { METZNER (1993); } \\
\text { METZNER \& MENZEL } \\
\text { (1996) }\end{array}$ \\
\hline & $\begin{array}{l}\text { Rotbuchen- } \\
\text { wälder }\end{array}$ & $\begin{array}{l}\text { Bradysia brevispina TUOMIKOSKI, 1960 } \\
\text { Bradysia confinis (WINNERTZ, 1867) } \\
\text { Bradysia fungicola (WINNERTZ, 1867) } \\
\text { Epidapus detriticola (KRATOCHVIL, 1936) } \\
\text { Epidapus lucifugus (MOHRIG, 1970) } \\
\text { Corynoptera abblanda FrEEMAN, 1983 } \\
\text { Corynoptera blanda (WINNERTZ, 1867) } \\
\text { Corynoptera cavipes MOHRIG, 1993 } \\
\text { Corynoptera perochaeta (MOHRIG \& MENZEL, 1990) } \\
\text { Corynoptera membranigera (KIEFFER, 1903) } \\
\text { Corynoptera minima (MEIGEN, 1818) } \\
\text { Corynoptera trepida (WINNERTZ, 1867) } \\
\text { Cratyna perplexa (WINNERTZ, 1867) } \\
\text { Ctenosciara lutea (MEIGEN, 1804) } \\
\text { Ctenosciara hyalipennis (MEIGEN, 1804) } \\
\text { Epidapus absconditus (VIMMER, 1926) } \\
\text { Epidapus atomarius (DE GEER, 1778) } \\
\text { Epidapus gracilis (WALKER, 1848) } \\
\text { Lycoriella lundstromi (FREY, 1948) } \\
\text { Lycoriella micria MOHRIG \& MENZEL, 1990 }\end{array}$ & $\begin{array}{l}\text { ALTMÜLlER (1976, } \\
\text { 1979); } \\
\text { DORN (1987); } \\
\text { HELLER (1990, 1996); } \\
\text { HÖVEMEYER (1985, } \\
\text { 1989b, 1992a, 1998); } \\
\text { HÖVEMEYER \& SCHAU- } \\
\text { ERMANN (2003); } \\
\text { IRMLER et al. (1996); } \\
\text { MENZEL et al. (2003); } \\
\text { SCHULZ (1996); } \\
\text { WARNING (1991) }\end{array}$ \\
\hline
\end{tabular}




\begin{tabular}{|c|c|c|c|}
\hline \multicolumn{2}{|c|}{ Biotop } & dominante Sciaridae-Arten & Literaturquellen \\
\hline \multirow[t]{6}{*}{ Laubwälder } & $\begin{array}{l}\text { Rotbuchen- } \\
\text { wälder }\end{array}$ & $\begin{array}{l}\text { Scatopsciara calamophila FreY, } 1948 \\
\text { Scatopsciara pusilla (MEIGEN, 1818) } \\
\text { Trichosia flavicoxa TuOMIKosKI, } 1960 \\
\text { Zygoneura sciarina MEIGEN, } 1830\end{array}$ & \\
\hline & $\begin{array}{l}\text { Rotbuchen- } \\
\text { Traubeneichen- } \\
\text { wälder }\end{array}$ & $\begin{array}{l}\text { Bradysia confinis (WINNERTZ, 1867) } \\
\text { Bradysia pectoralis (STAEGER, 1840) } \\
\text { Corynoptera membranigera (KIEFFER, 1903) } \\
\text { Ctenosciara hyalipennis (MEIGEN, 1804) } \\
\text { Epidapus detriticola (KRATOCHVIL, 1936) } \\
\text { Epidapus microthorax (BöRNER, 1903) }\end{array}$ & Schulz (1996) \\
\hline & $\begin{array}{l}\text { Rotbuchen- } \\
\text { Hainbuchen- } \\
\text { Traubeneichen- } \\
\text { Bergulmen- } \\
\text { Eschenwälder } \\
\end{array}$ & $\begin{array}{l}\text { Cratyna vagabunda (WINNERTz, 1867) } \\
\text { Ctenosciara hyalipennis (MEIGEN, 1804) }\end{array}$ & LEMBKe (1986) \\
\hline & $\begin{array}{l}\text { Hainbuchen- } \\
\text { Rotbuchen- } \\
\text { Eichenwälder }\end{array}$ & $\begin{array}{l}\text { Bradysia brevispina TuOMIKOSKI, } 1960 \\
\text { Bradysia longicubitalis (LENGERSDORF, 1924) } \\
\text { Corynoptera blanda (WINNERTZ, 1867) } \\
\text { Cratyna uliginosa (LENGERSDORF, 1929) } \\
\text { Ctenosciara hyalipennis (MEIGEN, 1804) }\end{array}$ & DrEws (1986) \\
\hline & $\begin{array}{l}\text { Stieleichen- } \\
\text { Hainbuchen- } \\
\text { wälder }\end{array}$ & $\begin{array}{l}\text { Corynoptera bistrispina (BUKOWSKI \& } \\
\text { LENGERSDORF, 1936) } \\
\text { Corynoptera forcipata (WINNERTZ, 1867) } \\
\text { Corynoptera luteofusca (BUKOWsKI \& } \\
\quad \text { LENGERSDORF, 1936) } \\
\text { Epidapus absconditus (VIMMER, 1926) } \\
\text { Epidapus atomarius (DE GEER, 1778) }\end{array}$ & HÖVEMEYER (1997) \\
\hline & $\begin{array}{l}\text { Stieleichen- } \\
\text { Hainbuchen- } \\
\text { Rotbuchen- } \\
\text { wälder }\end{array}$ & $\begin{array}{l}\text { Corynoptera membranigera (KIEFFER, 1903) } \\
\text { Ctenosciara hyalipennis (MEIGEN, 1804) } \\
\text { Epidapus absconditus (VIMMER, 1926) } \\
\text { Epidapus microthorax (BöRNER, 1903) }\end{array}$ & BOGENSCHÜTZ (2005) \\
\hline \multirow[t]{5}{*}{ Mischwälder } & $\begin{array}{l}\text { Fichten-Erlen- } \\
\text { Bergahorn- } \\
\text { Ebereschen- } \\
\text { Mischwälder }\end{array}$ & $\begin{array}{l}\text { Bradysia leucopeza MoHrig \& Mamaev, } 1989 \\
\text { Bradysia nitidicollis (MEIGEN, 1818) } \\
\text { Epidapus microthorax (BöRnER, 1903) }\end{array}$ & $\begin{array}{l}\text { SCHRANKEL (1998); } \\
\text { MENZEL (2006) }\end{array}$ \\
\hline & $\begin{array}{l}\text { Fichten- } \\
\text { Rotbuchen- } \\
\text { Mischwälder }\end{array}$ & $\begin{array}{l}\text { Ctenosciara hyalipennis (MeIgen, 1804) } \\
\text { Epidapus atomarius (De GeER, 1778) }\end{array}$ & Schulz (1996) \\
\hline & $\begin{array}{l}\text { Kiefern- } \\
\text { Stieleichen- } \\
\text { Mischwälder }\end{array}$ & $\begin{array}{l}\text { Bradysia brevispina Tuомıкоsкi, } 1960 \\
\text { Ctenosciara hyalipennis (MEIGEN, 1804) } \\
\text { Epidapus atomarius (De GeER, 1778) } \\
\text { Epidapus gracilis (WALKer, 1848) }\end{array}$ & $\begin{array}{l}\text { MOHRIG (1967); } \\
\text { SOUS-DORN \& DORN } \\
\quad(1990)\end{array}$ \\
\hline & $\begin{array}{l}\text { Kiefern-Trauben- } \\
\text { eichen-Rotbu- } \\
\text { chen-Hain- } \\
\text { buchen-Birken- } \\
\text { Mischwälder }\end{array}$ & $\begin{array}{l}\text { Corynoptera concinna (WINNERTZ 1867) } \\
\text { Cratyna nobilis (WINNERTZ, 1867) } \\
\text { Ctenosciara hyalipennis (MEIGEN, 1804) } \\
\text { Scatopsciara atomaria (ZETTERSTEDT, 1851) } \\
\end{array}$ & LEMBKe (1986) \\
\hline & $\begin{array}{l}\text { Traubeneichen- } \\
\text { Hainbuchen- } \\
\text { Lärchen-Linden- } \\
\text { Mischwälder }\end{array}$ & $\begin{array}{l}\text { Bradysia fungicola (WINNERTZ, 1867) } \\
\text { Cratyna vagabunda (WINNERTZ, 1867) }\end{array}$ & LeMBKe (1986) \\
\hline
\end{tabular}




\begin{tabular}{|c|c|c|c|}
\hline \multicolumn{2}{|c|}{ Biotop } & dominante Sciaridae-Arten & Literaturquellen \\
\hline \multirow[t]{3}{*}{ Nadelwälder } & $\begin{array}{l}\text { Kiefernwälder } \\
\text { (Reinbestand) }\end{array}$ & $\begin{array}{l}\text { Bradysia brevispina TuOMIKOSKI, } 1960 \\
\text { Corynoptera blanda (WINNERTZ, 1867) } \\
\text { Corynoptera globiformis (FREY, 1945) } \\
\text { Cratyna uliginosa (LENGERSDORF, 1929) } \\
\text { Ctenosciara hyalipennis (MEIGEN, 1804) } \\
\text { Epidapus atomarius (DE GEER, 1778) } \\
\text { Epidapus schillei (BöRNER, 1903) } \\
\text { Scatopsciara atomaria (ZETTERSTEDT, 1851) } \\
\text { Zygoneura sciarina MEIGEN, 1830 }\end{array}$ & $\begin{array}{l}\text { FELDMANN (1991, } \\
\text { 1992); } \\
\text { LEMBKE (1986); } \\
\text { MOHRIG (1967); } \\
\text { SOUS-DORN \& DORN } \\
\quad(1990)\end{array}$ \\
\hline & $\begin{array}{l}\text { Fichtenwälder } \\
\text { (Reinbestand) }\end{array}$ & $\begin{array}{l}\text { Corynoptera blanda (WINNERTZ, 1867) } \\
\text { Corynoptera luteofusca (BUKOWSKI \& } \\
\quad \text { LENGERSDORF, 1936) } \\
\text { Corynoptera membranigera (KIEFFER, 1903) } \\
\text { Cratyna uliginosa (LENGERSDORF, 1929) } \\
\text { Ctenosciara hyalipennis (MEIGEN, 1804) } \\
\text { Epidapus absconditus (VIMMER, 1926) } \\
\text { Epidapus alnicola (TUOMIKOSKI, 1957) } \\
\text { Epidapus atomarius (DE GEER, 1778) } \\
\text { Scatopsciara atomaria (ZETTERSTEDT, 1851) } \\
\text { Scythropochroa radialis LENGERSDORF, 1926 } \\
\text { Trichosia morio (FABRICIUS, 1794) }\end{array}$ & $\begin{array}{l}\text { DORN (1987); } \\
\text { HELLER (1990, 1996); } \\
\text { HOLSTEIN (1990); } \\
\text { HOLSTEIN \& FUNKE } \\
\quad \text { (1993); } \\
\text { IRMLER et al. (1996); } \\
\text { RudZINSKI (1989b, } \\
\text { 1992a); } \\
\text { THIEDE (1977); } \\
\text { WARNING (1991) }\end{array}$ \\
\hline & $\begin{array}{l}\text { Kiefern- } \\
\text { Fichtenwälder }\end{array}$ & $\begin{array}{l}\text { Bradysia brevispina TuMOMIKOSKI, 1960 } \\
\text { Corynoptera blanda (WINNERTZ, 1867) } \\
\text { Cratyna uliginosa (LENGERSDORF, 1929) } \\
\text { Ctenosciara hyalipennis (MEIGEN, 1804) } \\
\text { Scatopsciara atomaria (ZETTERSTEDT, 1851) } \\
\end{array}$ & DREWS (1986) \\
\hline \multicolumn{2}{|c|}{$\begin{array}{l}\text { Natur- und Kunsthöhlen } \\
\text { (incl. Bergwerkstollen) }\end{array}$} & $\begin{array}{l}\text { Bradysia forficulata (BEZZI, 1914) } \\
\text { Camptochaeta ofenkaulis (LENGERSDORF, 1925) } \\
\text { Corynoptera spoeckeri (LENGERSDORF, 1930) } \\
\text { Corynoptera subforcipata MOHRIG \& MENZEL, 1990 } \\
\text { Scatopsciara atomaria (ZETTERSTEDT, 1851) } \\
\text { Scatopsciara tenuicornis (LENGERSDORF, 1932) }\end{array}$ & $\begin{array}{l}\text { ECKERT et al. (1999); } \\
\text { MENZEL et al. (1990); } \\
\text { MENZEL \& MOHRIG } \\
\text { (2000); } \\
\text { MESSNER et al. (1982); } \\
\text { MoHrig \& MENZEL } \\
\text { (1993); } \\
\text { MoHrig et al. (1968); } \\
\text { WeBer (1991, 2001) }\end{array}$ \\
\hline
\end{tabular}

